\title{
Strengthening RTI/STD services: Lessons learned from a pilot project
}

Ismat Bhuiya

Population Council

Ubaidur Rob

Population Council

Follow this and additional works at: https://knowledgecommons.popcouncil.org/departments_sbsr-rh

Part of the Demography, Population, and Ecology Commons, Health Services Research Commons, and the International Public Health Commons How does access to this work benefit you? Let us know!

\section{Recommended Citation}

Bhuiya, Ismat and Ubaidur Rob. 1998. "Strengthening RTI/STD services: Lessons learned from a pilot project," Asia and the Near East OR/TA Project Final Report. Dhaka: Population Council. 


\title{
Strengthening RTI/STD Services in FP-MCH Program Lessons Learned from a Pilot Project
}

\author{
Final Report \\ Ismat Bhuiya \\ \& \\ Ubaidur Rob
}

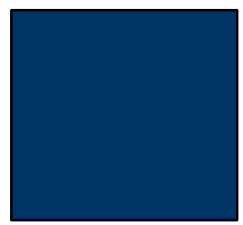

This project was supported by the Asia \& the Near East Operations Research and Technical Assistance project (ANE OR/TA) project of Population Council. The ANE OR/TA project is funded by the Office of Population, US Agency for International Development (USAID) funded the ANE OR/TA project through Contract No. DPE C-00-90-0002-10

Population Council

Dhaka, Bangladesh

May 1998 


\section{Table of Contents}

List of Tables

Page

List of abbreviations

$\mathbf{v}$

Acknowledgements

vi

Abstract

vii

ix

CHAPTER 1: Introduction 1

Background 1

MCH-FP Infrastructure 2

Health and Family Welfare Center 2

Staff Patterns $\quad 3$

Training $\quad 3$

Services $\quad 4$

Responsibilities $\quad 5$

$\begin{array}{ll}\text { Objective } & 7\end{array}$

Methodology $\quad 7$

Needs Assessment Phase $\quad 7$

Feasibility Study $\quad 7$

$\begin{array}{ll}\text { Baseline Survey } & 7\end{array}$

$\begin{array}{lr}\text { Intervention Phase } & 8\end{array}$

$\begin{array}{lr}\text { Evaluation Phase } & 9\end{array}$

CHAPTER 2: Needs Assessment 11

Results

$\begin{array}{ll}\text { Feasibility Study } & 11\end{array}$

Baseline Survey $\quad 11$

Observation of Service Delivery Process $\quad 11$

Questionnaire Survey $\quad 12$

Checklist Survey (Inventory) $\quad 17$

Observation of $\mathrm{H} \& \mathrm{FWCs} \quad 17$

CHAPTER 3: Intervention $\quad 21$

Components of the Intervention $\quad 21$

Preparatory Work for the Intervention $\quad 21$

$\begin{array}{ll}\text { Resource Mobilization } & 21\end{array}$

Distribution of Information Materials and Flow Charts 22

Developing Leaflet for Community

Developing a Checklist $\quad 22$

Readiness of H\&FWCs $\quad 22$

Establishing a Referral Mechanism

Intervention $\quad \mathbf{2 3}$

First Phase of Intervention $\quad 23$

Second Phase of Intervention $\quad 23$

History Taking $\quad 23$ 
Management of Client Flow $\quad 23$

Counseling $\quad 24$

Client Selection $\quad 24$

Reorganizing the FWV's Room 24

Third Phase of Intervention $\quad 25$

Refresher Training $\quad 26$

CHAPTER 4: Evaluation $\quad 31$

Knowledge Evaluation

Knowledge about RTI/STD

Knowledge about HIV/AIDS

Process Evaluation $\quad 32$

Client Flow

Client Flow Management

$\begin{array}{ll}\text { Client Privacy } & 33\end{array}$

History Taking $\quad 33$

Infection Prevention $\quad 34$

Examination $\quad 34$

Diagnosis and Treatment $\quad 34$

Type of Counseling Provided $\quad 35$

Partner Management $\quad 36$

Re-infection $\quad 36$

Referral Mechanism $\quad 36$

Assigned Day for Service Provision 36

CHAPTER 5: Client Profile $\quad 37$

$\begin{array}{ll}\text { Background Characteristic of the Clients } & 37\end{array}$

Reported Symptoms of RTI/STD Problems $\quad 37$

Prevalence of Reproductive Health Problems 38

CHAPTER 6: Lessons Learned and Policy Recommendations 39

References

Appendix 1: Checklist for the Observation of the Service Delivery Process 43

Appendix 2: Questionnaire for SACMO and FWV 46

Appendix 3:Questionnaire for FPI and FWA 51

Appendix 4: Checklist for Inventory $\quad 55$

Appendix 5: Leaflets for FWAs $\quad 58$

Appendix 6: Referral Form 59

Appendix 7: Syndromic Management Flow-Chart for Discharge (Bengali) 60

Appendix 8: Checklist for Client Profile

Appendix 9: Composition of DDS Kit and Satellite Kit 68

Appendix 10: Composition of New DDS kit 


\section{List of Tables}

CHAPTER 1:

Page

Table 1.1 Room Allocation for the Staff of the H\&FWC

2

\section{CHAPTER 2:}

Table 2.1 Distribution of H\&FWC Staff Members 12

Table 2.2 Availability of Medicine by H\&FWC 16

Table 2.3 Availability of Equipment by H\&FWC 16

$\begin{array}{lll}\text { Table 2.4 Availability of Supplies by H\&FWC } & 17\end{array}$

Table 2.5 Availability of Equipment and Supplies by H\&FWC 17

\section{CHAPTER 4:}

Table 4.1 Client Flow Before and After Intervention

Table 4.2 Distribution of Clients Who were Asked all of the Relevant Reproductive Health Questions Regarding Complaints of Discharge

Table 4.3 Percent Distribution of Clients for Whom Speculum was Used by Purpose of Examination $\quad 34$

Table 4.4 Diagnosis and Treatment of Diseases 35

Table 4.5 Percent Distribution of Clients by Type of Counseling Provided

\section{CHAPTER 5:}

Table 5.1

Table 5.2

Table 5.3
Background Characteristics of the Clients

Distribution of Clients by Reported RTI/STD Symptoms

Percent Distribution of Clients by Indications of Reproductive Health Problems 


\section{List of Abbreviations}

AIDS

ARI

ADCC

ADFP

ATFPO

BCG

DDFP

DDS

EPI

FP

FP-MCH

FPI

FWA

FWV

FWVTI

FPCST

GoB

H\&FWC

HIV

IUD

IP

MOHFW

MCWC

$\mathrm{MO}$ (MCH-FP)

MOs

$\mathrm{MCH}$

MOCC

MATS

MIP

$\mathrm{MC1}$

$\mathrm{MC} 2$

NIPORT

OT

PID

RTI

Sr. FWV

SACMO

STD

SSMC

TT

THC

TFPO

TBA
Acquired Immune Deficiency Syndrome

Acute Respiratory tract Infection

Assistant Director Clinical Contraceptives

Assistant Director Family Planning

Assistant Thana Family Planning Officer

Bacillus Calmette Guerin

Deputy Director Family Planning

Drugs and Dietary Supplement

Expanded Program on Immunization

Family Planning

Family Planning Maternal and Child Health

Family Planning Inspector

Family Welfare Assistant

Family Welfare Visitor

Family Welfare Visitor Training Institute

Family Planning Clinical Surveillance Team

Government of Bangladesh

Health and Family Welfare Center

Human Immunodeficiency Virus

Intrauterine Device

Infection Prevention

Ministry of Health and Family Welfare

Maternal and Child Welfare Center

Medical Officer Maternal Child Health and Family Planning

Medical Officers

Maternal and Child Health

Medical Officer Clinical Contraceptive

Medical Assistant Training School

Male Intervention Project

Mystery Client 1

Mystery Client 2

National Institute of Population, Research and Training

Operation Theater

Pelvic Inflammatory Disease

Reproductive Tract Infection

Senior Family Welfare Visitor

Sub Assistant Community Medical Officer

Sexually Transmitted Disease

Sir Salimullah Medical College

Tetanus Toxoid

Thana Health Complex

Thana Family Planning Officer

Traditional Birth Attendant 


\section{Acknowledgements}

This project was supported by the ANE OR/TA project of Population Council. The Office of Population, US Agency for International Development (USAID) funded the ANE OR/TA project through Contract No. DPE C-00-90-0002-10.

We take this opportunity to express our deepest gratitude to the Directorate of Family Planning for co-operation and support.

The authors also owe a great deal to a variety of persons. Of them, the support received from the following individuals deserves special mention. The individuals from the district level in the Government family planning programme and below were: Mr. Alfazuddin Ahmed, DDFP; Dr. Saidul Amin, ADCC; Dr. Faria Hasin, MOCC; Dr. Md. Makbul Hossain, MO (MCH-FP); and Mr. Sk. Saidur Rahman, TFPO of Tangail. Thanks are due to Dr. Saidul Amin, ADCC for carrying out the orientation session and identifying the resource person for hands-on training. Dr. Faria Hasin, MOCC took care of referral cases at the MCWC. Her inputs in the checklist for documenting client profile were also very helpful. Dr. Md. Makbul Hossain, MO (MCH-FP) and Mr. Sk. Saidur Rahman, TFPO helped us in mobilizing resources and in diffusing the message of availability of the RTI/STD services through the FWAs. We are also grateful to Dr. Md. Shamsuzzoha, National Medical Consultant, and Ms. Hosneara, Nurse Consultant, FPCST, for conducting orientation session for SACMOs and FWVs on RTI/STD/HIV/AIDS and hands-on training on infection prevention and RTI/STD case management.

We are also thankful to Sr. FWV, FWVs and SACMOs who participated in the project activities, and their hard work in rendering quality RTI/STD services and also for their valuable suggestions to make the program successful. The co-operation and enthusiasm of the FPIs and FWAs and the pharmacist was really appreciable.

We would like to acknowledge Mr. Nawab Ali, Ex. Director Administration, Directorate of Family Planning, for his support and co-operation. Special thanks go to Dr. Mizanur Rahman, Program Officer (Medical), AVSC International, for providing us Syndromic Management Chart and assistance on different technical issues. We are also grateful to Dr. Ahmed Al-Sabir, Director Research, and Mr. Subrata Kumar Bhadra, Statistician, NIPORT for providing us the FWV training manuals. Thanks are also due to Mr. Nitai Chakraborty, Associate Professor, Dhaka University for his assistance in data analysis and Dr. Zaman Ara, Assistant Professor, Sir Salimullah Medical College (SSMC), Midford for her assistance in finalizing the checklist for documenting the clients profile.

Finally let us express our gratitude to the administrative and support staff of Population Council, Dhaka for their untiring support in implementing the project. Special thanks go to Messrs. Abul Hossain Sarker, Mostak Ahmed, Abdul Majid, Nazimuddin Chowdhury, Abul Hossain and Sujan. The support from our colleagues Dr. Nahid Chowdhury, Program Coordinator and Dr. Sharif MI Hossain, Program Officer, during baseline survey also deserve special mention. 


\begin{abstract}
The national family planning and maternal child health (FP-MCH) program in Bangladesh has succeeded in raising family planning acceptance among the couples of reproductive age significantly. A major challenge still facing the program however, is to strengthen its reproductive health component. In view of this, Population Council launched a pilot project to examine the feasibility of strengthening comprehensive reproductive tract infection (RTI) and sexually transmitted disease (STD) services at the Health and Family Welfare Center (H\&FWC) level. The inputs from the Council included arrangement of training, provision of technical assistance in management, and other relevant supporting services required to strengthen the RTI/STD services.
\end{abstract}

The project had three phases. First, a needs assessment was conducted. Then the components of the intervention were identified, designed and implemented. And at the third phase, an evaluation was carried out. During the needs assessment phase, a feasibility study and a baseline survey were conducted. In the process, valuable experiences were gained with regard to RTI/STD service delivery at the primary level of the GoB service delivery system.

The needs assessment revealed that the service providers were aware of the importance of the RTI/STD problems and were willing to provide comprehensive RTI/STD services. The level of knowledge about RTI/STDs was poor among the service providers. History taking, clinical examination and counseling were practically nonexistent. Inquiry was only made about the reason of the visit; no attempt was made to discern the history of the condition, which is necessary for providing treatment of RTI/STDs. The choice of drugs for treating discharges was limited mostly to Metranidazole and in some instances, Ampicillin. In the system, there was no provision for drug for RTI/STD management. RTI/STDs were treated with the drug provided for other purposes. In some instances counseling on personal hygiene was made. No attention was given to partner management, compliance of drug prescription or follow-up.

There was no system to queue the clients. All of the clients crowded around the desk of the service providers. No use was made of the waiting room. The other findings included poor aseptic practices, indifference about privacy, and inadequate supply of materials essential for RTI/STD service delivery.

Much preparation was needed before the intervention. This included mobilization of resources from within the existing system; collection of information materials on RTI/STD; translation of an existing syndromic management flow-chart into Bangla; development of a checklist to record the clients' profiles; establishment of a referral mechanism for complicated cases; development of a leaflet in Bangla for FWAs and preparation at H\&FWCs for RTI/STD service delivey.

The intervention was done in three phases. The first phase included an orientation of the service providers on RTI/STD and hands-on training on how to prevent iatrogenic infections. In the second phase providers were given information about the importance of collecting an adequate history for each case and how to do so. Techniques of client management were discussed; the first come-first-serve system, with the help of a 'token' (a piece of paper with serial number) was introduced. Training on counseling was also provided. The components of counseling included compliance of drugs, partner management, condom use, follow-up visit and how to tailor counseling on specific symptom. Privacy was ensured by reorganizing the FWVs room. The third phase of the intervention was on RTI/STD case management. 
As a result of the intervention providers' knowledge about RTI/STDs improved. After the intervention the flow of clients with RTI/STD symptoms increased and client flow management improved with the introduction of the 'token' system. Privacy was strictly maintained during pelvic examination. This was not the case, however, with history taking. Service providers collected reproductive health information for more than 70 percent of the clients. Speculum examination was universal except for pregnancy cases. With regard to infection prevention, all of the providers were able to follow the infection prevention steps satisfactorily and confidently.

Speculum examination revealed that 98 percent of the clients had abnormal discharge. Of them, 83 percent had vaginal and 15 percent had cervical discharge. No cases with urethral discharge were found. Regarding the appropriateness of the treatments provided: none of the clients were given treatment for both gonorrhoea and chlamydia, even though 13 of the clients came with yellow cervical discharge and reported that their partner had urethral discharge or a genital ulcer. Six of the 13, however, were given a full course of drug required for the treatment of either gonorrhoea or chlamydia. The situation was much better for the treatment of trichomoniasis and bacterial vaginosis, and candidiasis. The type of counseling provided for prevention and management of RTI/STD varied. Issues relating to personal hygiene were discussed more often than partner treatment. Nearly half of the clients received counseling on condom use.

Drug compliance might have been high because only a very small number of re-infected clients came during the project period. Due to increased client flow, the program suffered from a shortage of essential consumable supplies like cotton, kerosene and detergent. Moreover, one female service provider was not adequate to serve the increased clients.

Findings suggest that the RTI/STD service component in the existing FP-MCH service delivery system can be strengthened without making any major changes in the program. 


\section{CHAPTER 1}

\section{Introduction}

\section{BACKGROUND}

The national family planning and maternal child health (FP-MCH) program in Bangladesh has made commendable progress in terms of raising family planning acceptance among the couples of reproductive age. An emerging challenge, however, is to integrate the reproductive health agenda within existing FP-MCH service delivery system. Thus, reproductive tract infections (RTIs) and sexually transmitted diseases (STDs) have a tremendous significance in the contex of the country's FP-MCH program. Clients at risk of pregnancy and in need of family planning services are same as the clients at risk of contracting RTI/STDs. Thus, symptoms of RTI/STDs, such as abnormal vaginal discharge, vaginal itching, and lower abdominal pain are often confused as the side-effects of certain family planning methods like IUD and injectables. The users of these methods often perceive the symptoms as contraceptive side effects and as a result, decide to discontinue family planning methods.

RTI/STDs include four types of infection, i) infection transmitted through sexual intercourse (STD); ii) infection resulting from an overgrowth of an organism normally present in the female genital tract of a healthy women (endogenous infection); iii) infection resulting from unhygienic medical procedures (iatrogenic infection); and iv) infection resulting from unhygienic health practices. These infections are preventable or treatable. But infections left untreated can have a profound impact on reproductive health. The consequences of these infections are infertility, ectopic pregnancy, cervical cancer, fetal wastage, low birth weight, infant blindness, neonatal pneumonia, and mental retardation. In addition, they facilitate the transmission of HIV.

RTIs and STDs are a growing concern in Bangladesh. From the limited information (Wasserheit et al., 1989; Chowdhury et al., 1995; Hussain et al., 1996), we know that more than half of the female population suffers from some kind of RTI. Studies on quality-of-care issues in the FP-MCH service delivery system (Barket et al., 1994; Rahman et al., 1996; Islam et al., 1996; Chowdhury et al., 1997) have consistently reported gaps in knowledge, breaches in infection prevention, negligence in the basic principle of history taking and clinical examination. Chowdhury et al. (1997) found that resources, such as trained staff, space, equipment and other facilities necessary for diagnosis and treatment of RTI/STDs are present in the existing FP-MCH service delivery system. Due to the lack of supportive supervision and the lack of initiative among the service providers, the issues of RTI/STD services have not been adequately addressed.

With the above in mind, Population Council launched a pilot project to examine the feasibility of strengthening comprehensive RTI/STD services at the Health and Family Welfare Center (H\&FWC) level. The project specifically aimed at assessing whether the current RTI/STD services at the H\&FWCs could be improved by motivating the service providers and utilizing the existing resources. The input from Population Council included training of the service providers on RTI/STDs, provision of technical assistance in management issues and coordination of the resources from the government $(\mathrm{GoB})$ system. 


\section{MCH-FP INFRASTRUCTURE}

The Directorate of Family Planning has six divisional directors. In each division, there are several districts with district-level family planning office headed by a deputy director (DDFP). At the district level there are also two assistant directors (AD); one for clinical contraception (ADCC) and the other for family planning (ADFP). There is provision for one medical officer clinical contraceptive (MOCC) at the district with more than eight thanas. The ADCC supervises the MOCC at district level as well as the medical officer, maternal child health and family planning (MO MCH-FP) at the thana level while the ADFP supervises the thana family planning officer (TFPO). The MOCC supervises senior family welfare visitors (Sr. FWVs) and family welfare visitors (FWVs) at the thana level (MCH unit), and the FWVs and the sub assistant community medical officers (SACMOs) at the union level (H\&FWC). In each thana there are one or two MO (MCH-FP), a medical officer for family welfare (MOFW), one TFPO, an assistant thana family planning officer (ATFPO), a Sr. FWV and a FWV. The MO (MCH-FP) supervises the staff of $\mathrm{MCH}$ unit at the thana level and the staff of H\&FWC at the union level. The Sr. FWV supervises the FWV at the thana level, and the FWVs and the SACMOs at the union level. She also assists the MO (MCH-FP) in sterilization. Where there are more than eight unions in a thana, there are two positions for MO (MCH-FP). In the district of Tangail there is a MOCC while in the Kalihati Thana there are two MO (MCH-FP).

\section{Health and Family Welfare Center $(H \& F W C)$}

The Health and Family Welfare Center (H\&FWC) is widely recognized as a primary level health care facility. There are two categories of H\&FWCs in terms of structure. The first one is one-storied building. At these centers, the service providers' residential

\begin{tabular}{|c|c|}
\hline \multicolumn{2}{|c|}{$\begin{array}{l}\text { Table } 1.1 \\
\text { Room Allocation for the Staff of the } \\
\text { Health and Family Welfare Center }\end{array}$} \\
\hline Staff & Furniture \\
\hline $\begin{array}{l}\text { Sub-Assistant Community } \\
\text { Medical Officer (SACMO) }\end{array}$ & $\begin{array}{l}1 \text { half secretariat table, } 4 \text { chairs, } 1 \\
\text { examination table and } 1 \text { stool for } \\
\text { peon }\end{array}$ \\
\hline Family Welfare Visitor (FWV) & $\begin{array}{l}1 \text { half secretariat table, } 4 \text { chairs, } \\
\text { and } 1 \text { stool for aya }\end{array}$ \\
\hline Family Planning Inspector (FPI) & 1 half secretariat table and 5 chairs \\
\hline IUD/ Operation Theatre & $\begin{array}{l}1 \text { examination table, } 1 \text { instrument } \\
\text { table and } 1 \text { almirah }\end{array}$ \\
\hline Recovery room & 2 beds \\
\hline $\begin{array}{l}\text { Health education/ Clients' seating } \\
\text { space }\end{array}$ & 7 long benches \\
\hline $\begin{array}{l}\text { Pharmacist (Drug store and drug } \\
\text { distribution room) }\end{array}$ & $\begin{array}{l}1 \text { half secretariat table, } 3 \text { chairs, } \\
\text { medicine dispensing table and } \\
\text { almirah }\end{array}$ \\
\hline
\end{tabular}
accommodation is behind the service center. The other is a two-storied building. Here the top floor is designated as the service providers' residential accommodation. In total, there are 3000 H\&FWCs. Of them, 246 have been upgraded. In the upgraded H\&FWCs, there are sterilization facilities, two position for FWVs and one position for a medical officer. In the entire system, there are 4,680 FWVs and 2300 SACMOs. Among the two facility categories, the first category sites were used for the intervention. According to the H\&FWC manual, the utilization of the rooms with a list of furniture is shown in the Table 1.1. 


\section{Layout of H\&FWCs (first category)}

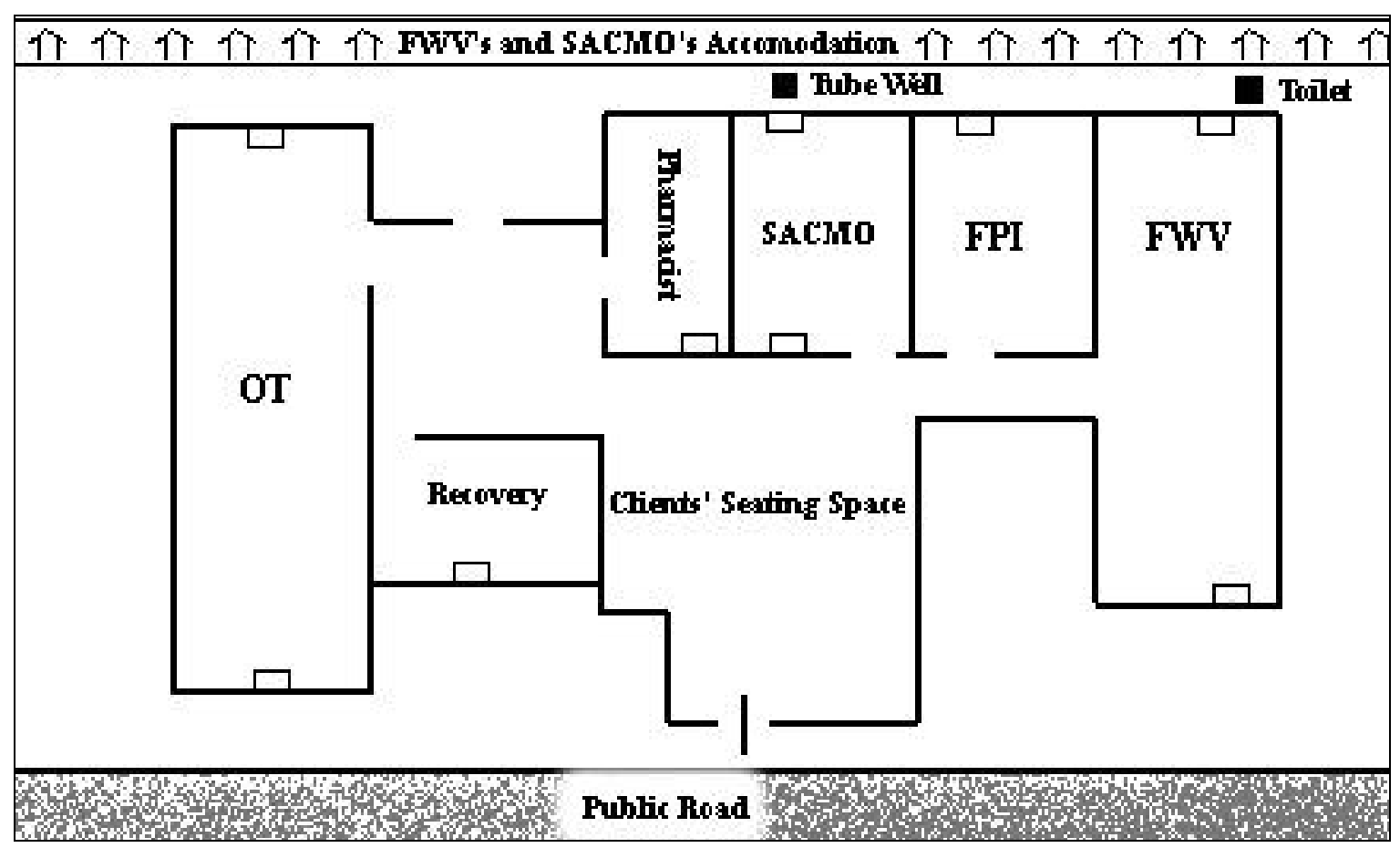

Source: Health and Family Welfare Center manual, 1986. MOHFW, Dhaka, Bangladesh

\section{Staff Patterns}

There are two types of staff member at the union level; one who works at the community level and the other who works at the clinic level. At the community level, there is a family planning inspector (FPI) and six to seven family welfare assistants (FWAs) who are responsible for motivating potential family planning (FP) clients. In addition to motivating clients, FWAs are responsible for distributing pills and condoms at the households' level. All of the FWAs are female, while all of the FPIs are male. The FPI supervises FWAs while FPIs are supervised by the TFPO. At the H\&FWC level, there is a SACMO, a FWV, a pharmacist, one female helper (ayah) and one night guard. All of the FWVs are female, while the SACMOs are mostly male. The MO (MCH-FP) at the thana level is the first line technical supervisor for the FWVs and SACMOs.

\section{Training}

The SACMO has ten years of schooling and three years of training from a Medical Assistant Training School (MATS). He/she also receives two weeks in-service training at Family Welfare Visitor Training Institute (FWVTI), which includes the following course curriculum: management, supervision, communication and counseling, coordination, responsibility and duty, staff management, service rules, logistics management, documentation, demography, family planning methods (all types), antenatal care, postnatal care, obstetric care, EPI, child growth monitoring, nutrition and prevention of vitamin A deficiency, prevention of iodine deficiency, primary health care, health education, respiratory disease, diarrhoea, skin diseases, intestinal parasite, RTI/STD/HIV/AIDS, minor ailments and infection prevention.

The FWV also has ten years of schooling and 18 months of training on--anatomy and physiology, pharmacology, communication, dirrhoea, EPI, ARI, child growth monitoring, 
nutrition deficiency disease, antenatal care, postnatal care, family planning, reproductive health and management of RTI/STD, First Aid, TBA training, management, satellite clinic and field training. After joining the service, FWVs receive two weeks of in-service training at FWVTI. The in-service training is based on the course curriculum of the basic 18-month training program.

\section{Services}

The H\&FWCs are entrusted to render both MCH and FP services. FWVs are not only providing services to the visiting clients in the H\&FWCs, but also providing services beyond the union center through satellite clinics.

The services which are provided by the H\&FWCs can be divided into two categories--namely routine service and special service. The routine services include:

- Health education

- Drinking water and intestinal worm prevention

- Prevention of diarrhoea and preparation of oral saline

- Care of pregnant mother and postnatal mother

- Family planning

- Child food and nutrition

- Personal and environmental cleanliness

- Prevention of night blindness

- Skin diseases

- Treatment

- Under-5 children

- Women of reproductive age (15-45)

- Other (men, the elderly)

- Services related to family planning methods

-FP services (except sterilization)

- Case referral

-Complicated cases are referred to the THC/distric hospital with a written prescription or a letter

- Organization of satellite clinic

- For those people who cannot come to the center for the service

- Nutrition education

- Ways to prepare nutritious food at low cost

- Improving food habits

- Ways to prepare food with maximum food value retention

Special services of the H\&FWC include:

- Involvement of traditional birth attendants

- FWVs organize training program for TBAs

- FWV organize meetings with TBAs every month

- FWV monitor the work of the trained TBAs

- EPI Day

- At the clinic

- In the field

- Meetings/Performance monitoring

- FWV and SACMO work periodically with FWAs in the field

- FWV and SACMO arrange monthly meetings with FWA 


\section{Responsibilities}

SACMO and FWV have two types of responsibilities. One is administrative, the other is professional. The SACMO is incharge of the H\&FWC and reports to the MO (MCH-FP).

Administrative responsibilities include:

- Supervising the staff of the H\&FWC

- Keep the H\&FWC neat and clean

- Maintain records and registers (11 record keeping registers)

- Collect reports from all staff member and send them to the appropriate authority

- Indent for necessary drugs, equipment and supplies

- Offering technical supervision to field staff (FWA and FPI) and arranging monthly meetings with them

- Supervising field staff and implementing their monthly activity plan

Professional responsibilities include:

- Diagnosis and treatment of diseases

- Provision of emergency treatment and First Aid

- Performance of small operations and dressing

- Provision of services to mothers and their children and assistance to the FWV with cases of sick children

- Support MO in sterilization camp

- Supervision of equipment sterilization

- Notification of higher authority of any epidemics in the locality

- Arrangement of regular health education sessions in the community

- Monitoring the community for potential epidemics, and prevent them if possible

- Follow-up on tuberculosis and leprosy cases at the clients' homes and provision of necessary treatment

- Maintenance of liaison with Union Parishad and Gram Committee, and seek their support in FP, sanitation and disease prevention activities

- Supervision of BCG vaccination activity, EPI, and vitamin A capsule distribution

The FWV reports to the MO, through the SACMO. Her administrative responsibilities include:

- Maintenance of infection prevention at H\&FWCs and satellite clinics

- Development of weekly plans for clinic day, satellite clinic and home visits

- Maintenance of record/preparation of reports

- Arrangement of meetings between FPI and FWAs

- Attendance at meeting called by the SACMO

Her professional responsibilities are as follows:

- Attend antenatal women; record pregnancy history, disease history, ensure TT vaccination, identify high-risk cases and refer them to THC and counsel the women about delivery

- Attend postnatal mother, examine uterus, detect any problems related to menstruation, examine newborn child, counsel the mother about FP methods

- Care for newborn children; register his/her weight, and treatment for illnesses

- Counsel mothers about child nutrition and vaccination

- Offer FP services to those who want to plan their families or limit their family size 
- Counsel potential clients about FP methods

- Assist the MO at the sterilization camp

- Conduct satellite clinics twice a week

- Make home visits for complicated cases referred by FWAs and TBAs

- Train TBAs to properly handle pregnant women

Pharmacist:

- Distribute medicine according to prescription written by MO (where there is an MO), SACMO, and FWV and explain the dose. He is also responsible for the cleanliness of the $\mathrm{H} \& \mathrm{FWC}$

- List necessary medicine and equipment in consultation with MO, SACMO and FWV every three months. It is his responsibility to ensure an adequate supply of medicine and equipment

- Maintain a register for medicine and equipment

- Assist SACMO in minor surgery and help in EPI activity

- Ensure proper storage of the medicine and supplies (some medicine have to be kept in the dark; some in the refrigerator)

- Perform simple laboratory tests

- Discard expired medicine

- Inspect linens and equipment at sterilization camp

\section{Equipment and Supplies}

The Ministry of Health and Family Welfare distributes six different types of kits to the H\&FWC. These include a drugs and dietary supplement kit (DDS kit), an MCH kit, an H\&FWC kit, a satellite kit, a midwivery kit and an IUD kit. The main components of the DDS kit and satellite clinic kit are drugs. Six DDS kits and four satellite clinic kits are supplied annually to all functional H\&FWCs. Beside these, there is often a selection of loose medicine available at the THC. The other four kits $(\mathrm{MCH}, \mathrm{H} \& \mathrm{FWC}$, Midwivery and IUD kit) are composed of necessary equipment and supplies need for service provision. All four kits are supplied once after the establishment of the H\&FWC. From June 1998, the government has introduced New DDS kit combining DDS and satellite clinic kits. Twelve new DDS kit will be supplied annually to each H\&FWC. The composition of the DDS, satellite kit and New DDS kit are attached in the Appendix 9 and Appendix 10. 


\section{OBJECTIVE}

The overall objective of the project was to develop a model of comprehensive RTI/STD service delivery at the primary level of GoB service delivery, located at the union level-the Health and Family Welfare Center (H\&FWC)

\section{Specific Objectives}

The specific objectives of the project were to:

- Assess the gaps in the current RTI/STD services at the H\&FWC level

- Determine the magnitude of the RTI/STD problem among the clients attending the H\&FWCs

- Introduce interventions to strengthen RTI/STD services

- Assess the change in RTI/STD client flow at the H\&FWCs due to the interventions

- Document the experience gained in implementing the RTI/STD interventions

\section{METHODOLOGY}

The project had three phases: needs assessment, intervention, and evaluation.

\section{Needs Assessment Phase}

In this phase, a feasibility study and a baseline survey was conducted.

\section{Feasibility Study}

The intervention sites were visited to assess the magnitude of the RTI/STD problem in the locality. This was done through discussions with MO in charge of the H\&FWCs, the FWVs, the SACMOs, the FPIs and the FWAs. Senior level medical personnel were also consulted about the possibility of launching such activities.

\section{Baseline Survey}

\section{Selection of the Site}

Elenga, Narandia, Sahadevpur and Paikara H\&FWCs under Kalihati Thana of Tangail District were selected purposively. The presence of a male intervention project (MIP) at these four centers and the knowledge of need for reproductive health care services beyond family planning which arose from the special counseling sessions for males/couples, prompted Population Council to use the male intervention platform for the RTI/STD intervention.

\section{Respondents and Information Collected}

Respondents included in the survey were SACMOs, FWVs, FPIs and FWAs of the four selected H\&FWCs, and the Sr. FWV at the Thana level.

In the baseline survey, information was collected on the willingness of the service providers to render RTI/STD services, the knowledge about RTI/STDs, the nature of current RTI/STD 
services, and the facilities currently available at the service delivery points for the management of RTI/STD.

\section{Survey Instruments}

Four sets of instruments were developed for data collection. These were:

- A checklist for the observation of the service delivery process

- A self-administered questionnaire for service providers, SACMO and FWV

- A self-administered questionnaire for FPIs and FWAs who work at the community level

- A checklist to inventory supplies and facilities

Questionnaires were pre-tested at two other H\&FWCs (Kawltia and Basan) in Gazipur Dstrict before finalization.

\section{Method of Data Collection}

The method of data collection included an observation of the service delivery process, conducting self-administered questionnaires and verifying the facilities.

First, a formal observation of the RTI/STD service delivery process was done. It was carried out with the help of two Mystery Clients (women who presented themselves as RTI/STDs' clients). Mystery Clients wished to remain anonymous. We will present them without their names for the purpose of the study. Of them, one was 22 years old and unmarried. The other was 26 years old and married. Each documented every step of service delivery she received from the H\&FWC. Before data collection, the two mystery clients were oriented on RTI/STDs and the essential elements of a quality RTI/STD service.

The second step of data collection was the self-administered questionnaire among SACMOs, FWVs, FPIs, and FWAs who were invited to H\&FWC for that purpose. An introduction was given about the aim of the project, the importance of the baseline survey and how to complete the questionnaire.

The third step of data collection was to document the available equipment, supplies and medicine relating to the management of RTI/STD problems and to verify the physical facilities, such as waiting space for clients, water source, facilities for pelvic examination, and privacy.

\section{Intervention Phase}

On the basis of the outcome of the baseline survey, the intervention package was developed. To minimize the workload of the service providers, the intervention was introduced in a phased manner. The orientation was interactive and the training included a demonstration with actual RTI/STD clients. The intervention started from October 1997.

There were three phases of the intervention:

\section{Phase One}

In the first phase, orientation about RTI/STDs was given, which included a definition of 
RTI/STDs, an explanation about the difference between RTI/STDs, an overview of the types of RTI/STDs, the common symptoms of RTI/STDs, the routes of transmission, means of prevention, consequences of RTI/STD, complications of RTI/STDs and the health behavior that influences the risk of RTI/STDs. The service providers were also given hands-on training on how to prevent iatrogenic infection.

\section{Phase Two}

In the second phase, hands-on training was given on how to greet clients, and how to take their history. The service providers were introduced to principles of counseling, maintenance of privacy and how to manage the client flow.

\section{Phase Three}

The third phase was on RTI/STD case management.

The first phase of the intervention was given to all of the providers of the four H\&FWCs. The second and third phases of intervention were introduced to only one center at a time. The first intervention was initiated at the Elenga. After one and a half months, the second center started the intervention as the same way as done in the Elenga. The newly-trained service providers from the first center with the help of a resource person initiated the second center's intervention. After four months, there was a refresher training session, comprising elements of all three phases. At that training session the remaining two centers were also included, so that they could all receive the training together and to see how far these two centers can render RTI/STD services without intensive training at their respective site.

The resource persons were identified from the government sector. The Sr. FWV was extensively used in second and third phases. For each center there was a special day for RTI/STD service delivery. A specially prepared leaflet was distributed through the FWAs to the community and directly to the clients who came to the center for other services. There were only three types of medicines available at the center that can be used for management of RTI/STDs. Clients were counseled properly about the non-availability of appropriate (prescribed) medicine. The complicated cases were referred to higher facilities.

\section{Evaluation Phase}

During the first week of April 1998, an evaluation was done on the knowledge of service providers and the process of service delivery. Data were collected from the self-administered questionnaire, which was used in the needs assessment phase. Since FPIs and FWAs were not used extensively in the program, an evaluation of their knowledge was not collected. The process evaluation was done only at Elenga and Narandia. 


\section{CHAPTER 2}

\section{Needs Assessment Phase}

\section{RESULTS}

\section{Feasibility Study}

All of the service providers felt that RTI/STDs were important health problems and they expressed their willingness to focus on that issue. One FWV reported having five to six abnormal discharge cases per month, while a SACMO reported having between nine to ten cases per month. The FWV and the SACMO both reported that their knowledge on this topic was not adequate enough to manage these cases. FWAs usually came across not only people with abnormal discharge, but also clients with genital itching, gonorrhoea and syphilis. The people of this group were mainly adolescents. They expressed their inability to handle the adolescents with RTI/STDs. The FPIs showed great interest in this activity and suggested that about 70 percent of the population in their catchment areas were suffering from abnormal discharge and genital itching.

Senior staff members of the FP-MCH program at the thana level were aware of the problems. They were concerned about the workload of service the providers and suggested the introduction of comprehensive RTI/STD services in a phased manner. By phasing the interventions in stages, the service providers would not consider the comprehensive RTI/STD services as additional ones and could render the services comfortably and efficiently.

\section{Baseline Survey}

\section{Observation of the Service Delivery Process}

Mystery client 1 (MC1) went to Elenga H\&FWC for treatment of vaginal itching. Many clients were in the service provider's (female SACMO) room. Naturally, MC1 felt uncomfortable about discussing her problems in front of the crowd. The provider explained to $\mathrm{MC1}$, "my room is always crowded like this. So you'll just have to talk about your problems in front of them". With great embarrassment, MC1 explained her problem to the provider. The provider neither took MC1's history in relation to the itching, nor did she examine the genital area. She simply told MC1, "this is due to an allergy. We do not have any medicine for your condition. Buy some Histacin (anti histamine) and take it, always use (wash the infected areas with) hot saline water". She wrote neither the name of the medicine nor the dosage for MC1.

MC1 learned of another service provider (FWV) and found no clients in her room. So MC1 entered the room and told the provider about her problem. The service provider asked MC1 what her name was. Then she wrote her (MC1) name. The FWV told MC1 to take a seat. The service provider wrote the name of a medicine on a piece of paper and went into another room. She returned with a liquid medicine (Gentian violet) and told MC1 to apply it to the infected area three times a day. She gave MC1 a piece of paper where she had written penicillin $250 \mathrm{mg}$, (28 tablets), to be taken one tablet four times a day and told her to buy the medicine from outside. Like the first service provider, the second also failed to take the client's history in relation to the vaginal itching. MC1 then wanted to use the washroom. So 
the provider said, "this is a real problem here. We do not have a washroom. You can use mine". Then she called an ayah and told her to take MC1 to her residence and show her the washroom.

Mystery client 2 (MC2) went to Narandia H\&FWC for the treatment of painful coitus. The provider did not take any history regarding the condition, which she termed as normal. She advised MC2 not to use any form of contraception before the birth of her first child.

\section{Questionnaire Survey}

\section{Staff Members of $H \& F W C s$}

Five FWVs, four SACMOs, four FPIs, 25 FWAs and one Sr. FWV from the thana level were

\begin{tabular}{|lccccc|}
\hline \multicolumn{5}{|c|}{ Table 2.1 } \\
& \multicolumn{5}{c|}{ Distribution of H\&FWC Staff Members } \\
\hline Catagory & Elenga & Narandia & Sahadevpur & Paikara & Total \\
\hline FWV & 2 & 1 & 1 & 1 & 5 \\
SACMO & 1 & 1 & 1 & 1 & 4 \\
FPI & 1 & 1 & 1 & 1 & 4 \\
FWA & 7 & 6 & 6 & 6 & 25 \\
\hline
\end{tabular}
the respondents of the baseline survey. Of the five FWVs and four SACMOs; two FWVs and one SACMO were from Elenga. Each of the other three centers have one FWV and one SACMO (Table 2.1). All of the FWVs had completed their basic training six to12 years and the SACMOs had completed their basic training 13 to 17 years back.

\section{Knowledge about RTI/STDs}

The SACMOs and FWVs were asked to define RTI/STD in writing. Respondents were also asked to name some common RTI/STDs, state the causes of RTI/STDs and describe the consequences of not treating RTI/STDs.

\section{$\underline{\text { SACMO }}$}

- Syphilis, gonorrhoea, trichonomiasis, moniliasis, pelvic inflammatory disease (PID) and AIDS were all mentioned as common RTI/STDs

- The SACMOs believed that RTI/STDs were caused by viruses and bacteria

- Only one SACMO mentioned that RTI/STDs could be transmitted through sexual intercourse between husband and wife

- They mentioned that untreated RTI/STDs could lead to impotency and infertility

\section{FWV}

- Syphilis, gonorrhoea, trichonomiasis, cervical erosion and PID were mentioned as common RTI/STDs

- Almost all the FWVs described RTI/STDs as a contagious disease

- A few of them explained that untreated RTI/STDs could cause infertility, death or cancer in the uterus

FPI and FWAs were asked whether or not they had heard, from their clients about abnormal vaginal discharge, vaginal itching or vaginal ulcers. They were then asked about the causes 
of such problems and how they could be prevented. Finally, they were asked whether those clients came to them for advice and if so, what advice they had given.

\section{$\underline{\text { FPI }}$}

- FPIs were aware of the symptoms of RTI/STDs

- They believed that lack of personal hygiene and intercourse during menstruation could cause genital itching or genital ulcer. For a burning sensation during micturation, they mentioned drinking insufficient water as a possible cause

- All of the FPIs said that a client could prevent RTI/STDs by consulting a doctor and advised the clients to visit a doctor

\section{$\underline{\text { FWA }}$}

- All of the FWAs were aware of the symptoms of RTI/STDs

- They reported that lack of knowledge regarding personal hygiene was the cause of RTI/STDs

- They also believed that by consulting a doctor, a client could prevent RTI/STDs. They similarly advised the RTI/STD clients to visit a doctor

\section{Knowledge about HIV/AIDS}

SACMOs and FWVs were asked whether or not they had heard about a disease called AIDS, whether or not they were aware of the routes of transmission of HIV/AIDS, how could it be prevented and whether it is curable.

\section{$\underline{\text { SACMO }}$}

- All of the SACMOs had heard about a disease called AIDS

- Most of them believed that sexual intercourse, sex with a person with AIDS, reuse of a contaminated syringe-needle and poor personal hygiene were responsible

- Most of them reported that the use of disposable syringe, use of condom, avoidance of sex with person with AIDS, and maintenance of personal hygiene were means of prevention

- All of them suggested that there was no cure for AIDS

- None of the SACMOs mentioned that transfusion of infected blood, vertical transmission from mother to child in the womb and breast feeding could also be responsible

- None of them suggested that transfusion of screened blood and avoiding birth by infected mother could also be a means of prevention

\section{FWV}

- All of the FWVs had heard about a disease called AIDS

- Some of the FWVs believed that reuse of contaminated syringe-needle, sex with a person with AIDS, and poor personal hygiene were ways of contracting AIDS

- A few of them mentioned use of a disposable syringe and maintenance of good personal hygiene as the means of prevention

- Almost half of the FWVs believed that there was no cure for AIDS 
- None of the FWVs mentioned that transfusion of unscreened blood, vertical transmission from mother to child in the womb and breast feeding could also be responsible

- None of the FWVs suggested that the use of a condom or avoidance of high risk sexual behavior, use of only screened blood for transfusion, and avoiding birth by infected mother could also be means of prevention

FPIs and FWAs were asked whether they had heard about a disease called AIDS, whether or not they were aware of the routes of transmission of HIV/AIDS, and how it could be prevented.

\section{FPI}

- All of them had heard about a disease called AIDS

- Most of them knew that AIDS was caused by having multiple sex partners

- A few of them suggested that the avoidance of high-risk sexual behavior could prevent AIDS

\section{$\underline{\text { FWA }}$}

- All of them had heard about a disease called AIDS

- A few of them reported that AIDS was caused by having multiple sex partners, having sex with a person living with AIDS, and having frequent intercourse

- Nearly half of the FWAs believed that the use of a condom was a means for preventing AIDS. A few mentioned that avoidance of multiple sex partners, avoidance of sex with persons living with AIDS and maintenance of personal hygiene could prevent AIDS

\section{Type of RTI/STDs Symptoms}

SACMOs and FWVs were asked which type of RTI/STD clients they had encountered and which age groups they belonged to.

\section{$\underline{\text { SACMO }}$}

- SACMOs had come across almost all types of RTI/STDs symptoms

- Most of the SACMOs reported these problems as being more prevalent among men and women older than 20 years

- Some of them were familiar with the symptoms of both males and females RTI/STDs

\section{FWV}

- FWVs had come across all types of RTI/STD symptoms

- Some of them reported these problems as being more prevalent among men and women older than 20 years

- One FWV was most familiar with RTI/STD symptoms

FPIs and FWAs were asked which type of RTI/STD problems they encountered in their practice and which age group suffered from these problems. 
- FPIs reported that they had come across clients with abnormal discharge, lower abdominal pain, a burning sensation during micturation, discharge from the penis, pain in testes, swelling in the groin and itching genitalia

- All of them mentioned these problems were more prevalent among men and women older than 20 years

\section{$\underline{\text { FWA }}$}

- The most common symptoms they had come across were abnormal discharge, lower abdominal pain, burning sensation during micturation, and a discharge from the penis

- Almost all of the FWAs had encountered various types of RTI/STD among both male and female clients

- The FWAs also mentioned that these problems were more prevalent among men and women older than 20 years

\section{Management of RTI/STDs}

SACMOs and FWVs were asked about the treatment and advice for RTI/STD symptoms.

\section{$\underline{\text { SACMO }}$}

- SACMOs provided curative services as well as preventive advice

- Most of them were more familiar in managing female RTI/STDs than male symptoms

- The common symptoms managed by the SACMOs were abnormal discharge, lower abdominal pain, vaginal ulcer, vaginal itching, ulcerated genitalia (male and female), burning sensation during micturation (female) and pussy discharge from penis. These symptoms were managed without any history taking or examination

- SACMOs usually prescribed medicine available at the H\&FWC along with preventive advice

- Sometimes the treatment was not specific. For instance, for all discharge cases, the SACMOs provided metronidazole. In some cases, they provided ampicillin instead of doxycyclines (ampicillin is not the drug of choice). According to their statements, doxycyclines were not available at the H\&FWCs

- They offered advice only for two symptoms--abnormal discharge and burning sensation during micturation. For abnormal discharge, they advised practice of good personal hygiene and use warm saline water to wash the genitalia. In case of burning sensation during micturation, they advised the client to drink plenty of water

- One SACMO claimed that she usually performs local examination and treats both husband and wife together. Upon verification, this claim was proven false

- None of the SACMOs mentioned condom use, compliance of medicine and follow-up in his or her advice

$\underline{\text { FWV }}$

- FWVs provided curative services as well as preventive advice

- FWVs were more familiar with managing female RTI/STDs than male's ones 
- The common symptoms managed by the FWVs were abnormal discharge, lower abdominal pain, vaginal ulcer, vaginal itching, burning sensation during micturation (female), painful coitus, and pussy discharge from penis

- Usually prescribed medicine available at the H\&FWC along with preventive advice

- Sometimes the treatment was not specific. For instance, for all the discharge cases, the FWVs provided metronidazole. In some instances, they provided ampicillin instead of doxycycline (ampicillin is not the drug of choice). According to their statements, they had managed the cases with the drugs available at the H\&FWC

- The two FWVs claimed that they usually performed local examination. Upon verification, this claim was proven false

- Except in lower abdominal pain and painful coitus, they offered advice for all of the symptoms. For abnormal discharge and vaginal ulcer, they advised practice of good personal hygiene and use of warm saline water to wash the genitalia; for vaginal itching, use of warm saline water; for ulcer in female genitalia, pussy discharge from penis, they advised the practice of good personal hygiene. In case of burning sensation during micturation, they advised the client to drink plenty of water

- None of the FWVs mentioned condom use, partner treatment, compliance of medicine and follow-up check up in her advice to the client

\section{Drugs for RTI/STDs}

Service providers were asked about the types of medicine they are supplied from the government for RTI/STD treatment. They stated that in reality, they were not getting any

\begin{tabular}{|lcccc|}
\hline \multicolumn{5}{|c|}{ Table 2.2} \\
Availability of Medicine by H\&FWC \\
\hline Medicine & Elenga & Narandia & Sahavedpur & Paikara \\
\hline Cap. Ampicillin & $*$ & $*$ & NA & NA \\
Tab. Metronidazole & $*$ & $*$ & $*$ & $*$ \\
Tab. Nystatin & $*$ & $*$ & $*$ & $*$ \\
Tab. Cotrimoxazole & $*$ & $*$ & NA & NA \\
Tab. Hysomide & $*$ & $*$ & NA & NA \\
Gentian Violet & $*$ & $*$ & $*$ & $*$ \\
\hline
\end{tabular}

* Indicates availability of drugs

NA Indicates non availability of drugs

Table 2.3

Availability of Equipment by H\&FWCs

\begin{tabular}{lcccc|}
\hline Equipment & Elenga & Narandia & Sahadevpur & Paikara \\
\hline Sim's speculum & $*$ & $*$ & $*$ & $*$ \\
Cuscos speculum & $*$ & $*$ & $*$ & NA \\
Sponge holding forcep & $*$ & $*$ & $*$ & $*$ \\
Gully pot & $*$ & NA & NA & NA \\
Instrument tray & $*$ & $*$ & $*$ & NA \\
Lifter & $*$ & $*$ & $*$ & NA \\
Spot light/torch light & $*$ & $*$ & $*$ & $*$ \\
Autoclave & $*$ & NA & NA & NA \\
Brush & $*$ & $*$ & NA & NA \\
Bucket & $*$ & $*$ & NA & NA \\
Strainer & NA & NA & NA & NA \\
Stirrer & NA & NA & NA & NA \\
Measuring cup & NA & NA & NA & NA \\
Sterilizer (boiling) & $*$ & $*$ & $*$ & $*$ \\
Stove & $*$ & $*$ & $*$ & $*$ \\
Thermometer & $*$ & NA & $*$ & $*$ \\
\hline
\end{tabular}

$* \quad$ Indicates availability of equipment

NA Indicates non availability of equipment medicine specifically for RTI/STD treatment. Thus they were rendering RTI/STD services with the medicines provided for other purposes. Two SACMOs and three FWVs mentioned that the supply of medicine was not adequate to meet the needs of the clients (Table 2.2).

\section{Equipment for RTI/STD Services}

Service providers were asked about the availability of the equipment that was needed for the provision of RTI/STD services. Elenga had all of the necessary equipment for pelvic examination while other centers did not have a gullypot. Autoclave was not available at the three centers but these centers did have stoves and sterilizer (IUD set sterilizer which was also used for boiling other instruments). Strainer, stirrer and measuring cup for bleaching powder were not available at four centers. The 
buckets and brushes were not available at two centers. Thermometer was not available at one center (Table 2.3).

\section{Supplies for RTI/STD Services}

Bleaching powder and detergent powder were not available at the H\&FWCs. The H\&FWCs

\begin{tabular}{|lcccc|}
\hline \multicolumn{5}{c|}{ Table 2.4 } \\
\hline \multicolumn{5}{c|}{ Availability of Supplies by H\&FWC } \\
\hline Supplies & Elenga & Narandia & Sahavebpur & Paikara \\
\hline Bleaching powder & NA & NA & NA & NA \\
Powder detergent & NA & NA & NA & NA \\
Bar soap & NA & $*$ & NA & $*$ \\
Gloves & $*$ & NA & $*$ & $*$ \\
Cotton & $*$ & $*$ & $*$ & $*$ \\
Dettol/savlon & $*$ & NA & NA & NA \\
Spirit & $*$ & NA & $*$ & $*$ \\
Chlorohexidine/hibisol & $*$ & $*$ & $*$ & $*$ \\
$* \quad$ Indicates availability of supplies & & & \\
NA Indicates non availability of supplies & & & \\
\hline
\end{tabular}
at Narandia and Paikara had bar soap, while those at Elenga and Sahadevpur did not. These supplies are crucial for infection prevention. Cotton was available at all centers. According to the service providers, gloves were not available only at Narandia. Dettol/savlon was not available at three centers (Table 2.4).

\section{Checklist Survey (Inventory)}

An inventory was conducted at four H\&FWCs. It was found that some of the items reported as not available during the needs assessment, were available at the time of inventory. This

\begin{tabular}{|lcccc|}
\hline \multicolumn{5}{|c|}{ Table 2.5} \\
Evailability of Equipment and Supplies by H\&FWCs \\
\hline Equipment-supplies & Elenga & Narandia & Sahadevpur & Paikara \\
\hline Simuipment & & & & \\
Cuscos speculum & $*$ & $*$ & $*$ & $*$ \\
Sponge holding forcep & $*$ & $*$ & $*$ & NA \\
Gully pot & $*$ & $*$ & $*$ & $*$ \\
Instrument tray & $*$ & $*$ & NA & $*$ \\
Lifter & $*$ & $*$ & $*$ & $*$ \\
Spot light/torch light & $*$ & $*$ & $*$ & NA \\
Autoclave & $*$ & NA & NA & NA \\
Brush & $*$ & $*$ & $*$ & $*$ \\
Bucket & $*$ & $*$ & $*$ & $*$ \\
Strainer & $*$ & $*$ & $*$ & $*$ \\
Stirrer & NA & NA & NA & NA \\
Sterilizer (boiling) & $*$ & $*$ & $*$ & $*$ \\
Stove & $*$ & $*$ & $*$ & $*$ \\
Thermometer & $*$ & NA & $*$ & $*$ \\
Supplies & & & & \\
Bleaching powder & NA & NA & NA & NA \\
Powder detergent & NA & NA & NA & NA \\
Bar soap & $*$ & $*$ & $*$ & $*$ \\
Gloves & $*$ & NA & $*$ & $*$ \\
Cotton & $*$ & $*$ & $*$ & $*$ \\
Dettol/savlon & $*$ & NA & NA & NA \\
Spirit & $*$ & NA & $*$ & $*$ \\
Chlorohexidine/hibisol & $*$ & $*$ & $*$ & $*$ \\
$*$ Indicates availability of supplies & & & \\
NA Indicates non availability of equipment-supplies & & \\
& & & & $*$ \\
\hline
\end{tabular}
implies that the service providers were ignorant about the equipment and supplies in their respective centers (Table 2.5).

\section{Observation of H\&FWCs}

\section{Waiting Space}

The open space in the middle had a few benches where clients could sit and wait. But instead of sitting there, all of the clients crowded in the service providers' rooms. Thus, there was no privacy. Moreover, there was no initiative taken by the service providers to control the client flow in to their rooms.

\section{Sitting Arrangement of Service Providers}

In the $\mathrm{H} \& \mathrm{FWC}$ manual there is no requirement for an examination table for FWVs (H\&FWC manual, 1986). By rearranging examination table at the clinics, however, the FWV was able to get an examination table (the one that was designated for the SACMO). At Elenga, the FWV had a pelvic examination table of her own while the SACMO had a simple examination table. The SACMO at Elenga is female. She used her examination table for general examination. When necessary, she used the FWV's 
examination table for antenatal and postnatal check-ups. At the upgraded Elenga H\&FWC, two FWVs are required to share a room. The OT is used for sterilization, an activity which takes place once every fortnight. The recovery room is used for autoclaving (sterilizing) the instruments. The FPI uses his room only when he comes to the office (once a week).

\section{Layout of Elenga H\&FWC}

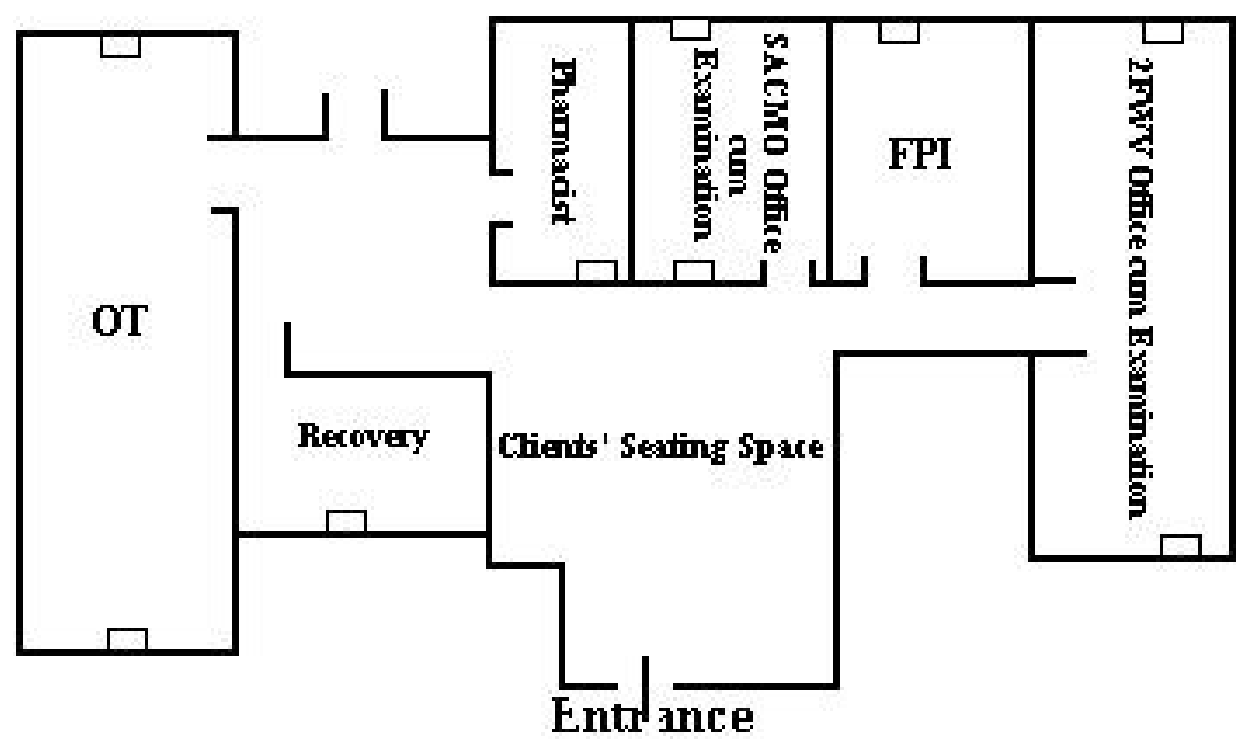

Layout of Narandia, Sahadevpur and Paikara

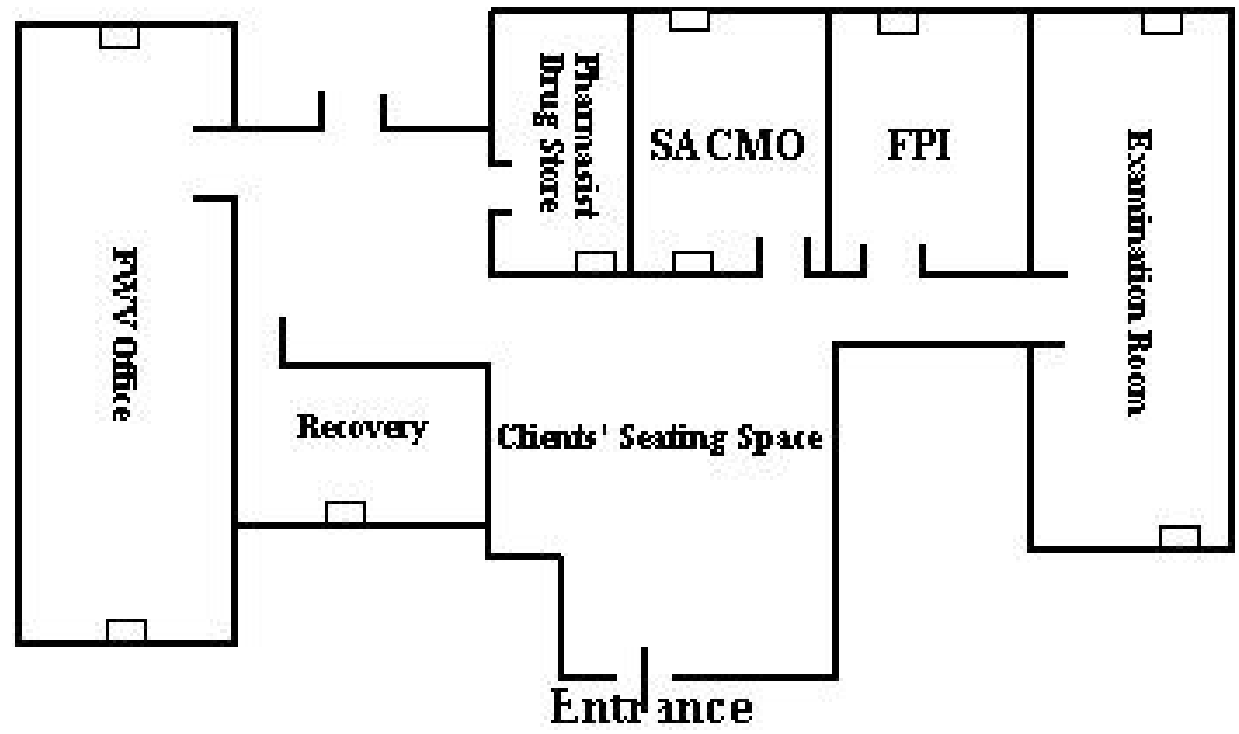

Male and female sterilization facilities are only available at the upgraded H\&FWCs. OT room is sometimes used for delivery at non-upgraded H\&FWCs. At Narandia, Sahadevpur and Paikara, the pelvic examination room and the FWV's office room (sitting room) are separate. Infact, their office room and examination room are situated at opposite ends of the building. The FWVs use their designated room for examination and the OT room as an office. At each of these facilities, there is one general examination table in the FWV's room and another table of same kind in the other room. The second is used for pelvic examination. The recovery room is not utilized. 


\section{Client Privacy}

All the four H\&FWCs are situated very close to the public road (layout of H\&FWC at page 3). There was a lack of privacy in both the FWV's and the SACMO's room. This lack of privacy stemmed from two primary factors: first, the clients gather in service providers' room. Secondly, the examination table is situated next to the window for maximum light during an examination. In Elenga, a toilet stands a few yards away from this particular window. In Narandia, there is a public road on the other side of the window. These windows had a thin curtain, which was not sufficient to maintain the privacy of the clients. Same situation exists in Sahadevpur and Paikara. During examination, if someone goes to the toilet or passes along the road, the client on the examination table really becomes tense tries to cover herself. Under such a circumstance, it is difficult for the service providers to examine the client properly. There was a curtain hung around the pelvic examination at Elenga. There was no curtain around the examination table at Narandia, Sahadevpur and Paikara.

\section{Drug Stores}

The drug stores at the H\&FWCs are not organized for optimal functioning. The products were stored in a haphazard manner. There was no system of indicating products that should be used first to avoid expiration and wastage.

\section{Disposal of Waste}

The clinical waste was thrown in the open space a few yards away from the service center. There was no system of burning or burying the waste.

\section{Water Supply}

There were tube-wells at the back of all the service centers. The service providers used the water stored in a bucket for washing their hand, which was collected by ayah from the tubewell. 


\section{CHAPTER 3}

\section{Intervention}

\section{COMPONENTS OF THE INTERVENTION}

Intervention components were as follows:

- Knowledge about RTI/STDs

- Infection prevention

- Reorganization of the FWVs room

- History taking

- Assurance of privacy

- Management of client flow

- Clinical examination

- Diagnosis of RTI/STDs by using a syndromic management chart

- Treatment of RTI/STDs by using standard treatment regime

- Counseling including partner management and follow-up

- Optimal organization of drug stores

- Training of FWAs to disseminate the message of RTI/STD service availability

\section{PREPARATORY WORK FOR THE INTERVENTION}

Much preparation was needed before the intervention. Following is a description of these activities.

\section{Resource Mobilization}

There were several meetings with DDFP, ADCC, MO (MCH-FP) and TFPO of Tangail. The meetings were interactive and the participants' expressed their willingness to undertake the activities. The main discussion was focused on imparting knowledge on RTI/STD, hands-on training on infection prevention (IP), availability of necessary supplies, RTI/STD diagnosis, treatment, supply of medicine and establishing a referral system for complicated cases. At the meeting, it was agreed that for RTI/STD services, IP should be introduced during the first intervention phase. The MO (MCH-FP) took the responsibility of ensuring the supply of bleaching powder, and all other necessary supplies. The ADCC took the responsibility for giving the orientation on RTI/STD; identifying resource persons for hands-on training on IP, how to examine clients and how to diagnose the problems. The ADCC suggested to utilize the FPCST team (the team usually monitor the quality of services provided from the $\mathrm{H} \& \mathrm{FWCs}$ ) as resource team. He expressed his willingness to contact the FPCST team.

The MO (MCH-FP) and TFPO also took responsibility of disseminating the message of RTI/STD service availability through the FWAs. Diffusion of the message of the availability of the RTI/STD services would naturally be contingent upon the readiness of each H\&FWC to render the comprehensive RTI/STD services. The MO (MCH-FP) added that for ease of service delivery, RTI/STD services should be rendered once a week. The ADCC suggested the need for penicillin injection (high power) from the government. He also pointed out the necessity of a Bengali version of the standard RTI/STD treatment regime for FWVs and SACMOs. The MO (MCH-FP) agreed to help the ADCC to conduct the orientation session 
on RTI/STD. It was decided that the first intervention would first be implemented at Elenga, then at Narandia, followed by the other centers. At these hands-on training sessions, the trainees were Sr. FWV, SACMOs and FWVs.

To approve the periodic utilization of the FPCST team, Population Council approached the FPCST team leader.

\section{Distribution of Information Materials and Flow Charts}

Bangla materials on RTI/STD, flow charts on syndromic management and infection prevention charts contributed by various organizations were distributed among the trainers and providers. The flow charts for discharge were translated into Bangla for the purposes of the intervention.

\section{Developing Leaflet for Community}

A leaflet in Bangla with information about RTI/STD symptoms and the treatments available at the FWC was developed and distributed among the FWAs. The FWAs were instructed to inform the community about the services.

\section{Developing a Checklist}

A checklist for recording the clients' profile was developed. The FWVs and SACMOs were trained to complete the checklist. The checklist has several sections covering information such as socio-demographic, relevant history, examination, diagnosis and drug prescription, counseling offered and follow-up conducted.

\section{Readiness of $\mathrm{H} \& \mathrm{FWCS}$}

The H\&FWCs were made ready for RTI/STD intervention one after another in terms of logistics. First, Elenga H\&FWC was prepared. Each H\&FWC was considered ready when six or more speculums, a reasonable number of gloves, and an adequate quantity of bleaching powder was available. Bleaching powder and gloves were supplied by the MO (MCH-FP) from the Thana Health Complex (THC).

\section{Establishing a Referral Mechanism}

Establishment of a referral mechanism was done through discussions with district-level and thana-level officers. It was agreed that clients with complications could be referred to Maternal and Child Welfare Center (MCWC), THC and District Hospital. For testing of specimen for complicated cases, the doctors would use private laboratories in that area for emergency purposes, for the time being. Otherwise, the RTI/STD cases would be managed on the basis of clinical signs and symptoms. A referral form was developed and used to refer clients to the upper-level facilities. Referral compliance was verified with the referral centers after two to three weeks.

After every consultation with the FPCST team, the time and date of hands-on training was fixed. 


\section{INTERVENTION}

The intervention was implemented in three phases.

\section{First Phase of Intervention}

In the first phase, orientation was given to both the SACMOs and the FWVs of the four H\&FWCs. Orientation was given by ADCC and FPCST staff. Population Council staff described the importance of the RTI/STD services and maintenance of high quality. The ADCC gave an overview of the diseases. The FPCST team provided orientation on RTI/STD/HIV/AIDS. The orientation on RTI/STD/HIV/AIDS included topics on symptoms of RTI/STD and HIV/AIDS, routes of transmission, preventive measures, consequences of RTI/STDs and the syndromic management of RTI/STDs. The FPCST team also provided hands-on training on how to prevent iatrogenic infections. This was done through a extensive demonstration, which was later repeated by each service provider in the presence of the trainer.

\section{Second Phase of Intervention}

During second phase, hands-on training was provided by Population Council staff in one of the H\&FWCs at a time.

\section{History Taking}

The procedure of history taking emphasized rapport building before asking questions related to the problems. The service providers were instructed to greet the clients first and then to make queries about the major complaint and other related problems. In case of abnormal discharge, the clients were asked about the duration of the problems, color, odor, and amount of discharge. They were also asked about the presence of pain in the lower abdomen, current contraceptive use, and pregnancy history. Other related problems, like itching genitalia, pain in passing urine and pain during coitus were also covered. Inquiries were also made about the client's husband; whether he had experienced a pussy discharge, pain, ulcer, itching or warts in the genital area. Information on his nature of work and usual place of residence was also collected during history taking.

\section{Management of Client Flow}

For efficient client management, system of first-come-first-serve was introduced with the help of a token. Client was given a piece of paper with a serial number on it (token) and told her to take a seat and wait to be called by the service provider. To ensure the client's privacy, only the client who came for services could enter the service provider's room. Then the door of the room was secured. It was found that this system of client flow management not only maximized privacy but also improved the quality of the service delivery.

Orientation was also given to the clients regarding the new system of service delivery. With regard to expected waiting room behavior, their cooperation was also sought. The clients' seating arrangement was rearranged so that they could sit more comfortably. Moreover, orientation on the importance of the maintenance of privacy was given to those clients who came on the training day at the H\&FWCs. 


\section{Counseling}

With regard to counseling, the first four elements (Four Cs) of counseling were taught. These were COMPLIANCE of medicine, CONTACT tracing or partner management, CONDOM use and COME BACK for a follow-up check up. Hands-on training was also given on how to tailor the counseling to be relevant to the disease(s) diagnosed. Observation was also done during counseling and any weaknesses noticed were corrected on the spot.

\section{Client Selection}

Clients were selected on a priority basis. Other than on the RTI/STD service delivery day, pregnant women got first priority, followed by injection clients who came for subsequent doses. Other clients and RTI/STD clients were giving lowest priority. It was observed that one RTI/STD client requires 30 minutes for total service delivery. It was very difficult for the service providers to manage RTI/STD clients along with other services. The service providers tried to convince the clients either to come on RTI/STD service delivery day or to wait until they were finished with all of the other clients.

On RTI/STD service delivery day, clients were asked (when they received the token from the ayah) for their reason for visiting the H\&FWC. On that day, RTI/STD clients got first priority (after pregnant women if there were any). All of non-RTI/STD clients were advised to return to the center on another day for services. The other clients who could receive services on RTI/STD service delivery day included those with contraceptive complications, pregnant women and serious general patients.

\section{Reorganizing the FWV's Room}

Reorganizing the FWVs room at Elenga was a little more difficult than it was at the other centers because the OT at Elenga used for male and female sterilization. The FWVs and the SACMO of the respective center had a discussion with their FPI about the rearrangement of the rooms. The FPIs agreed to give their rooms to the FWVs. It was further agreed that FPIs would use recovery room if required. In Narandia, Sahadevpur and Paikara there were no sterilization facilities. Thus the recovery room was assigned to the FPIs.

In the reorganization process, two centers have a separate examination room, and a separate room for the FWV to take the clients' history and provide counseling. These two rooms are adjacent to one another. Both the FWVs and the SACMO were using the examination room for examination purposes. Since there were two FWVs at Elenga, and were using the same room, one FWV would take history while the other did her examination. One staff member from Population Council helped them to designate the use of the rooms, organize the drug store and manage client flow. 
Layout of the Elenga H\&FWC after the intervention

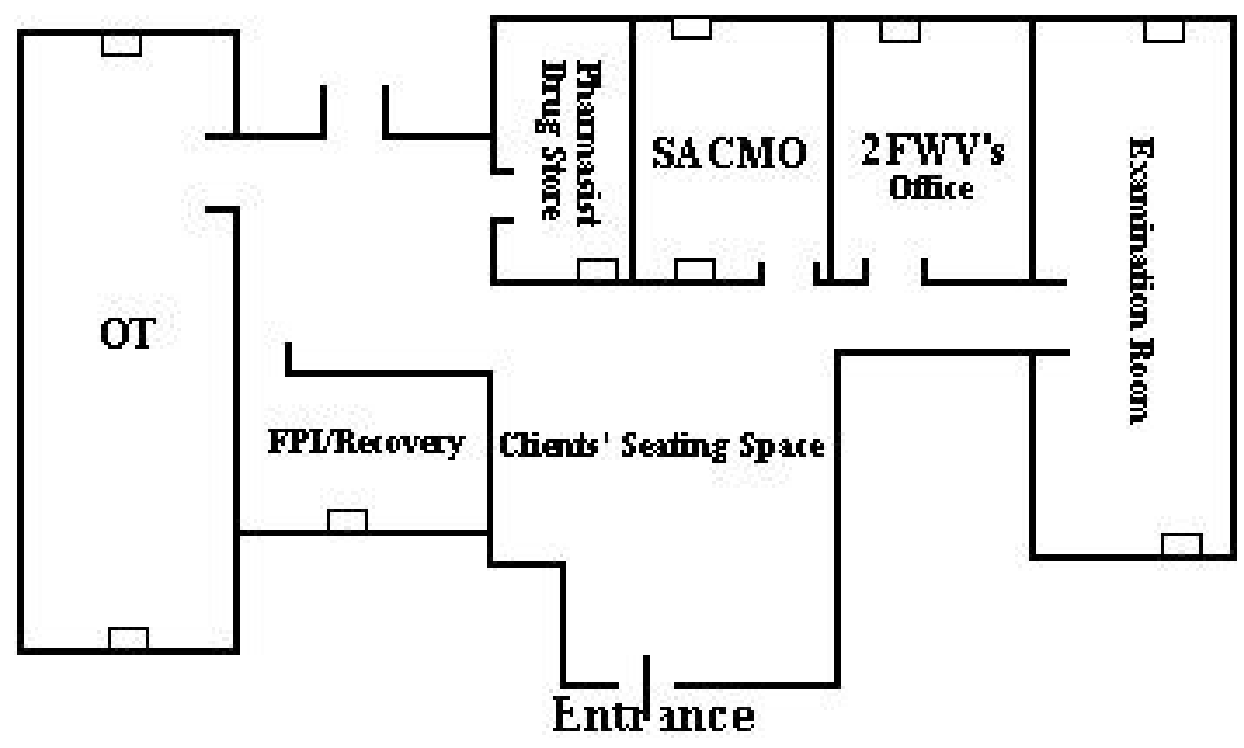

Layout of the Narandia, Sahadevpur and Paikara H\&FWC after the intervention

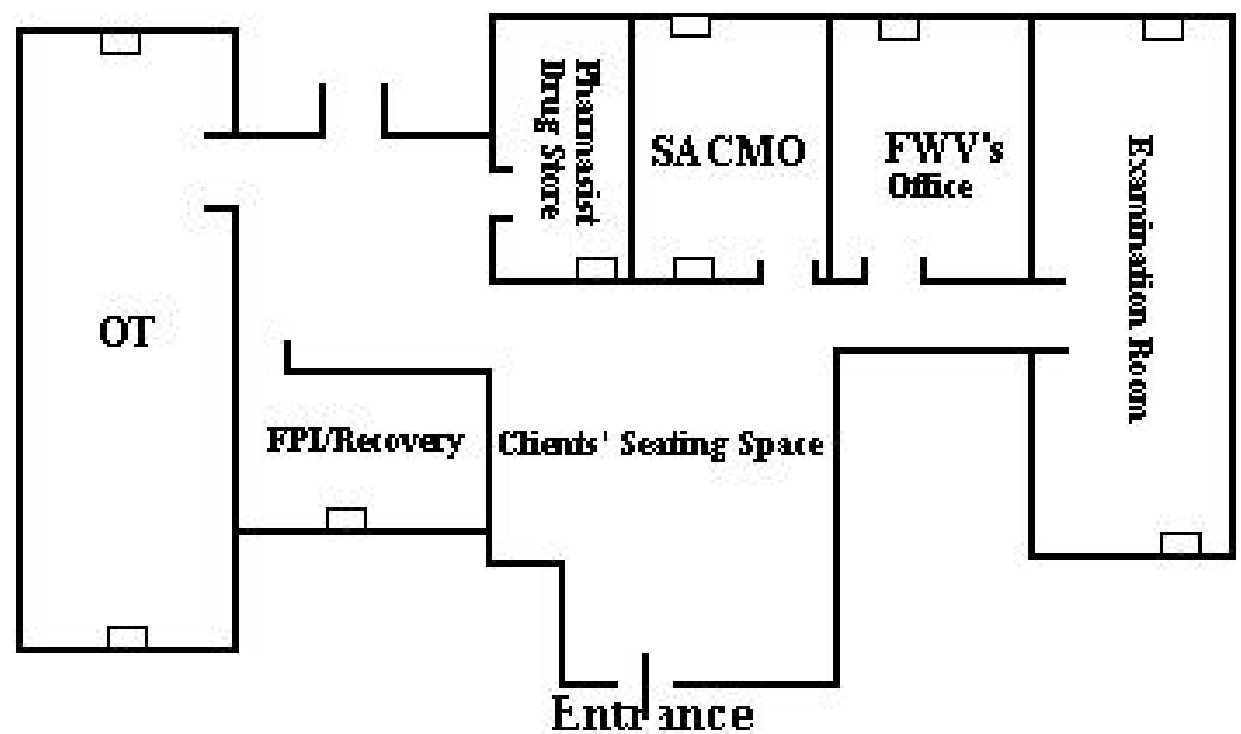

\section{Third Phase of the Intervention}

The third phase of the intervention was on the management of RTI/STDs. This activity included: sterilization of instruments, preparation of decontamination solution, management of client flow, client selection, history taking, examination, diagnosis, treatment and counseling. Before commencing the third phase, the FWAs of the respective H\&FWCs were asked to disseminate the message that RTI/STD services were available every Thursday at Elenga beginning on 16 October 1997 and every Sunday at Narandia beginning on 30 November 1997. 


\section{Elenga}

On Thursday 16 October 1997, the FWVs and SACMOs first made the decontamination solution and then sterilized the speculum set under the supervision of the FPCST team. Clients collected their tokens and waited for services in the designated area. SACMO and FWVs both took the history. The FPCST nurse demonstrated each step of a standard examination for the female SACMO, FWVs and Sr. FWV with the first client. Medicine was prescribed with the help of a syndromic management chart. The client was counseled on hygienic practice, compliance of the medicine, partner treatment, condom use and returning to the clinic for follow-up. The Sr. FWV handled the next client. After that, the female SACMO and the FWVs examined the remaining clients in the presence of the FPCST team. That day, 12 clients came for RTI/STD services. Thus, each service provider got about three opportunities to provide comprehensive RTI/STD services. Among the 12 clients, two were referred to the MCWC. Thereafter, Population Council staff monitored the newly-educated providers from time to time. The monitoring was done through direct observation and examining the checklist that was used for documenting the clients' profile.

\section{Narandia}

Approximately about two months after the training at Elenga, on Sunday 30 November 1997, Narandia was started the phase three intervention as the same fashion as done in Elenga. The Elenga FWV also participated in the training with the Narandia FWV. The Sr. FWV first initiated the process under the supervision of the FPCST team. FWV from Elenga examined the second case and Sr. FWV managed the third case. After these three cases, the Narandia FWV was given the opportunity to examine the fourth case. That day, Narandia FWV managed five cases. The FPCST team, along with the Population Council staff, supervised the whole process. Fourteen clients came for RTI/STD services on that day. Among the 14 clients three were referred to the MCWC. After the training, Narandia service providers were monitored periodically in the same way as was done for the Elenga staff.

\section{Refresher Training}

A repetition of the training in relation to the three phases mentioned above was done four months after the initial training. Refresher training was arranged at Sahadevpur on February 1998. Unfortunately, the FWV from Sahadevpur could not attend the training. Newlytrained staff from two of the participating H\&FWCs along with the Paikara staff were present at the training. On that day, all three steps of the intervention were reinforced. Three trained FWVs and one trained SACMO from the Elenga and Narandia H\&FWCs initiated the process and the FPCST team, along with Population Council staff, observed the process. Nineteen clients came for RTI/STD services on that day. Among them, three were pregnant and one was menstruating. Among the remaining 15 clients, one was referred to the MCWC. 
Orientation Session 


\section{CHAPTER 4}

\section{Evaluation}

Evaluation was done in two areas: knowledge of service providers and proficiency of service delivery. The knowledge evaluation was done through a self-administered questionnaire conducted during the needs assessment phase. The process evaluation was conducted at the centers where RTI/STD services had been rendered for 3-4 months.

\section{KNOWLEDGE EVALUATION}

\section{Knowledge about RTI/STD}

\section{SACMO}

- In addition to the common RTI/STDs mentioned in the baseline survey (syphilis, gonorrhoea, trichonomiasis, moniliasis/candidiasis, PID and AIDS), the SACMOs were able to mention chlamydia

- In comparison to the causes mentioned in the baseline survey (virus and bacteria), the SACMOs reported that personal hygiene, sexual contact with infected person and infection passes from mother to child (congenital) were the causes of RTI/STDs

- In addition to the consequences listed for leaving RTI/STDs untreated, in the baseline survey (infertility), the SACMOs reported cancer of the cervix, disease in the nervous system and abortion

\section{FWV}

- In addition to the common RTI/STDs mentioned in the baseline survey (syphilis, gonorrhoea, trichonomiasis, cervical erosion and PID), the FWVs were able to mention white discharge, lower abdominal pain, vaginal itching and chlamydia

- In addition to the consequences listed for leaving RTI/STDs untreated, in the baseline survey (infertility, death and cancer in the uterus), the FWVs mentioned abortion and pain during coitus

\section{Knowledge about HIV/AIDS}

\section{SACMO}

- In comparison to the causes mentioned in the baseline survey (sexual intercourse, sex with a person with AIDS and reuse of a contaminated needles and syringes), the SACMOs now believed that AIDS could be contracted through the use of unsterile instruments, from infected mother to child, and through blood transfusion

- In comparison to the correct means of prevention listed in the baseline survey (use of disposable syringe, use of condom, avoidance of sex with person with AIDS), the SACMOs now mentioned that use sterile instruments, sex with a faithful partner and maintenance of personal hygiene could be the means for prevention

- None of the SACMOs mentioned breast feeding could also be responsible 
- None of them reported transfusion of only screened blood and avoidance of birth by infected mothers as being means of prevention. One SACMO still believed that improper personal hygiene could cause AIDS

\section{FWV}

- In comparison to the correct responses given in the baseline survey (reuse of contaminated syringe-needle and sex with a person with AIDS), the FWVs now reported that AIDS could be contracted through sex with a prostitute, sex with more than one partner, blood transfusion and use of unsterile instruments

- In comparison to the correct responses given in the baseline survey ( use of a disposable syringe), the FWVs now mentioned the use of a condom, use of sterile instruments, use of sterile gloves, and avoidance of multiple sex partners as the means of prevention

- None of the FWVs believed that there was a cure for AIDS

- The FWVs still did not mention that vertical transmission from mother to child in the womb and breast feeding could also be responsible for contracting AIDS. Nor did they mention transfusion of screened blood and avoidance of birth by infected mother as means of prevention

\section{PROCESS EVALUATION}

\section{Client Flow}

The intervention was implemented during a different time period for each of the H\&FWCs. Elenga had been providing RTI/STD services beginning in October 1997 while Narandia began in December 1997. As the RTI/STD services quality was improved, the flow of clients

\begin{tabular}{|lcccc|}
\hline \multicolumn{5}{|c|}{ Table 4.1 } \\
\multicolumn{4}{|c|}{ Client Flow Before and After Intervention } \\
\hline \multicolumn{4}{c}{ Before Intervention } & \multicolumn{1}{c|}{ After intervention } \\
\hline Elenga & Jan '97-Sept '97 & AMCF & Period & AMCF \\
Narandia & Jan '97- Nov'97 & 10 & Oct '97-Mar'98 & 23 \\
\hline AMCF & Average monthly client flow & Dec-Feb'98 & 12 \\
\hline
\end{tabular}
with RTI/STD symptoms increased at both centers (Table 4.1). It seemed that the center with all female service providers drew more clients than the one with male and female providers. It was observed that female clients were reluctant to report discharge to a male provider. This implied that female clients preferred female service providers for RTI/STD services. Regarding client flow there could have been seasonal effect. For example, there were no clients in March in one center. It was also found that due to an increased client flow, the centers reported a shortage of cotton. The leaflet used by the FWAs at the community level provided information on availability of RTI/STD services worked well.

\section{Client Flow Management}

Introduction of the token system improved client flow management. This system worked better at Narandia than at the Elenga. In Narandia, the night guard works in the daytime and was able to help ayah manage client flow. 


\section{Client Privacy}

It was observed that privacy was strictly maintained during pelvic examination. This was not the case with history taking.

\section{History Taking}

Service providers collected reproductive health information for more than 70 percent of the clients. Eight pieces of information were to be collected from the clients with regard to their menstrual history. These were i) whether the menstrual period was regular or irregular, ii)

\begin{tabular}{|lc|}
\hline \multicolumn{2}{|c|}{$\begin{array}{c}\text { Table } 4.2 \\
\text { Distribution of Clients Who were Asked all of the Relevant Reproductive } \\
\text { Health Questions Regarding Complaints of Discharge }\end{array}$} \\
\hline Reproductive health questions & Percent \\
\hline Menstrual history (8 items) & 74.4 \\
Obstetric history (6 items) & 83.0 \\
History of abnormal discharge (4 and above items) & 90.9 \\
History of other related problems (14 items) & 70.0 \\
History of husband's problem (9 items) & 77.0 \\
\hline $\mathrm{N}$ & 176 \\
\hline
\end{tabular}

age of menarche, iii) last menstrual period, iv) frequency of menstrual period, v) length of menstrual period, vi) how many times a day the clients had to change their menstrual pad during menstruation, vii) whether there was any pain in lower abdomen during menstruation and viii) whether there was a foul smell to their menstrual blood. Seventy four percent of the clients were asked all eight questions (Table 4.2).

In case of obstetric history, six questions to be asked were, i) age at marriage, ii) age at first childbirth, iii) number of living children, iv) nature of last delivery, v) place of last delivery and vi) the provider for the last delivery. Eighty three percent of the clients were asked all six questions (Table 4.2).

Regarding abnormal vaginal discharge, following six questions were to be asked: i) how long the clients has had the problem, ii) frequency of discharge, iii) amount of discharge, iv) color of discharge, v) smell of discharge and vi) consistency of discharge. None of the providers asked all six questions. However, for 91 percent of the clients, information on more than three out of six items was collected (Table 4.2).

Twelve pieces of information were to be collected from the clients with regard to other related problems. These included i) whether there was any genital itchiness, ii) whether there had been any genital ulceration during the last three months iii) and if there had been, did they secrete pus or blood, iv) whether there was pain during coitus, v) whether there had been pain in lower abdomen during the last three months, and if so vi) what was the nature of the pain; vii) whether there had been any problem in passing urine, viii) frequency of passing urine, ix) whether they had any dysentery, $x$ ) whether the client had sought any treatment, if so xi) from whom and xii) when. It was found that 70 percent of the clients were asked 14 questions. The client's current contraceptive use was also recorded (Table 4.2).

Regarding husband's history, nine questions were supposed to be asked. These included i) whether there were any problems in the husband's genitalia, if so ii) was there any itching, iii) ulcer, iv) pain, v) pus, vi) discharge from penis, vii) whether there were any warts in genitalia, viii) whether the problems had been treated, if so ix) by whom. Providers asked 77 percent of the clients all of the above questions (Table 4.2).

It was observed that in the case of the female service providers, the clients were proactive and 
the client provider-interaction was spontaneous. At the Narandia H\&FWC a male service provider took the history while the female service provider did the examination. It was found that in some cases the history did not match the examination findings. It was observed that the client did not mention all symptoms to the male provider. In some cases, even with female service providers, there was some miss-match between the history and the examination findings. This was either because the provider had not successfully elicited the accurate history or the client had not presented her problem properly.

\section{Infection Prevention}

All of the providers knew how to make the decontamination solution (5 percent chlorine solution) and how to dip the instruments into the solution. The ayah also knew how to clean the instruments. The providers were more comfortable with sterilizing the instruments by boiling than with autoclaving. After the intervention, all of the providers could successfully carry out the infection prevention steps.

\section{Examination}

The examination included palpation of lower abdomen for tenderness and inspection of genital area (without instruments) for discoloration, inflammation, swelling, nodules, ulcers and discharge. All of the clients were examined for lower abdomen and genital area.

\section{Speculum Examination}

Except the pregnancy cases, all of the clients were examined with a speculum. This allowed

\begin{tabular}{|lc|}
\hline \multicolumn{2}{|c|}{$\begin{array}{c}\text { Table 4.3 } \\
\text { Percent Distribution of Clients for Whom Speculum was } \\
\text { Used by Purpose of Examination }\end{array}$} \\
\hline Purpose of examination & Percent \\
\hline Existence of discharge & 98.3 \\
Color of discharge & 96.0 \\
Amount of discharge & 95.5 \\
Odor of discharge & 92.6 \\
Condition of the cervix & 94.9 \\
All of the above elements examined & $\mathbf{8 8 . 6}$ \\
\hline $\mathrm{N}$ & 176 \\
\hline
\end{tabular}

the service provider to check the condition of the cervix, the origin of the discharge, its color, and odor, and whether the discharge was profuse, scanty or moderate. With the help of the speculum the service providers examined 98 percent of the clients for existence of the discharge; 96 percent for the color of the discharge; 95 percent for the amount; and 93 percent for the odor of the discharge. The provider examined the condition of the cervix for 95 percent of the clients. Approximately 89 percent of the clients were examined for all the five elements (Table 4.3).

\section{Bimanual Examination}

Bimanual examination was done for cervical motion and tenderness, fornix tenderness and adhesion, and Bartholin gland tenderness. Except pregnancy cases, all of the clients were examined for the above elements of bimanual examination.

\section{Diagnosis and Treatment}

Table 4.4 shows the extent to which the providers treat the clients appropriately. The treatment regime was considered correct if it followed the standard one suggested in syndromic management manual. Of the 13 who came with yellow cervical discharge and 
reporting that their partner had urethral discharge or genital ulcer (Table 5.3), none were given treatment for both gonorrhoea and chlamydia. Six of the 13 (46\%), however, were given the full course required for the treatment of either gonorrhoea or chlamydia. The situation was much better for trichomoniasis or bacterial vaginosis, and candidiasis. In case of trichomoniasis and bacterial vaginosis, the clients who came with watery, smelly discharge; or frothy, smelly discharge; or profuse, watery, smelly discharge; or profuse, frothy, smelly discharge was considered for trichomoniasis and bacterial vaginosis treatment. It was found that in 77 percent (10/13) of the cases of trichomoniasis and bacterial vaginosis, providers treated the condition correctly. Twenty five percent (44 clients) of the clients had none of the above symptoms (Table 4.4).

\begin{tabular}{|lcc|}
\hline \multicolumn{3}{|c|}{ Dable 4.4 } \\
& \multicolumn{2}{|c|}{ Diagnosis and Treatment of Diseases } \\
\hline Diagnosis & Percent received correct treatment & $\mathrm{N}$ \\
\hline Gonorrhoea \& Chlamydia & 46.2 & 13 \\
Trichomoniasis \& & 77.0 & 13 \\
Bacterial Vaginosis & & \\
Candidiasis & 82.3 & 113 \\
\hline $\mathrm{N}$ & & 176 \\
\hline Multiple responses were possible & \\
\hline
\end{tabular}

Some of the clients had other infections like ulcer, lower abdominal pain and itching along with the above symptoms. These clients were prescribed or provided with additional medicines along with those prescribed for their main symptoms. These additional medicines were metronidazole, gentian violet and nystatin which are available in the H\&FWC pharmacy. Metronidazole was used in $11(85 \%)$ of the 13 cases of symptoms of gonorrhoea and chlamydia. Generally, the providers provided available medicine from the H\&FWC pharmacy and prescribed an antibiotic from outside (buy from the market).

It was observed that with these limited supply of drugs (metronidazole, gentian violet and nystatin), the providers were trying to treat RTI/STDs cases. This implied that the treatment was not specific in all the cases. Providing medicine available at the H\&FWC pharmacy became part of the service providers' habit. It was thus, more comfortable for them to prescribe only those medicines they could provide. It was learned that if the provider counseled the client about the severity of the disease and the medicine available at the $\mathrm{H} \& \mathrm{FWC}$ was not appropriate for that disease, they were willing to buy the medicine from the market.

\section{Type of Counseling Provided}

The service providers counseled approximately 96 percent of the clients. More than twothirds of the clients had received counseling on personal hygiene, more than half on

\begin{tabular}{|lc|}
\hline \multicolumn{2}{|c|}{$\begin{array}{c}\text { Table 4.5 } \\
\text { Percent Distribution of Clients by Type of Counseling Provided }\end{array}$} \\
\hline Elements of Counseling & Percent \\
\hline Wash genitalia with warm saline water & 70.0 \\
Use clean, dry cloth for menstrual protection & 66.5 \\
Complete full course of medicine even if symptoms & 52.9 \\
disappear & \\
Use condom until cured & 49.4 \\
Come back for follow-up checkup after 3/7 days & 59.4 \\
Use vaginal suppository, following instructions carefully & 34.1 \\
Both partners have to take medicine at the same time & 11.2 \\
\hline N & 170 \\
\hline
\end{tabular}
compliance of medicine and follow-up checkup. Regarding partner management, about half of the clients had received counseling on condom use and 11 percent on partners' treatment. In connection to the appropriateness of counseling, it was found that two percent who had received counseling on personal hygiene, ten percent who had received counseling on condom use and five percent on partner's treatment--which was not required. 


\section{Partner Management}

Partner management was done in one of three ways, and in combination with counseling. The three ways included 1) providing medicine for the partner, 2) prescribing medicine for the partner and 3) advising the client to bring the partner to the clinic.

\section{Re-infection}

The program did not have the opportunity to follow-up the cases at the community level. Therefore, one cannot be certain about the compliance of the prescription and medical advice. In fact, the compliance might have been high because a very small number of re-infected clients ( 7 clients) came for treatment during the project period.

\section{Referral Mechanism}

The other aspect of the intervention was the referral system, which worked satisfactorily. During the referral process, the clients were given the choice as to where they preferred to go. All of the six referred clients expressed their preference in going to the MCWC. Of the six referred clients, five went to seek help from the referral centers.

\section{Assigned Day for RTI/STD Service Provision}

The clients who recognized their RTI/STD symptoms were attending H\&FWC on the assigned day of the week for RTI/STD services. But the providers were also getting RTI/STD symptoms in other clients who came for antenatal, postnatal and FP services. Thus, every day RTI/STD services were provided. 


\section{CHAPTER 5}

\section{Client Profile}

\section{BACKGROUND CHARACTERISTICS OF THE CLIENTS}

One-hundred-and-seventy-six clients were provided RTI/STD services at Elenga and

\begin{tabular}{|lc|}
\hline \multicolumn{2}{|c|}{ Background Characteristic of the Clients } \\
\hline Category & Percent \\
\hline Age & 3.0 \\
$>20$ & 21.3 \\
$20-24$ & 30.8 \\
$25-29$ & 21.9 \\
$30-34$ & 23.0 \\
$35+$ & $\mathbf{1 0 0 . 0}$ \\
Total & 27.0 \\
Mean & 28.0 \\
Median & 169 \\
\hline N & \\
\hline Education & 51.2 \\
Illiterate & 31.0 \\
Completed primary & 17.8 \\
Primary and above & $\mathbf{1 0 0 . 0}$ \\
Total & 174 \\
\hline N & \\
\hline Husband's Education & 47.3 \\
Illiterate & 17.8 \\
Completed primary & 25.4 \\
Completed secondary & 9.5 \\
Secondary and above & $\mathbf{1 0 0 . 0}$ \\
Total & 169 \\
\hline N & \\
\hline Husband's Occupation & 35.8 \\
Agriculture & 33.3 \\
Business & 18.2 \\
Service & 12.7 \\
Transport worker & $\mathbf{1 0 0 . 0}$ \\
Total & 165 \\
\hline N & \\
\hline &
\end{tabular}

Narandia. Among the total 176 clients, 98 percent were married and two percent were unmarried adolescents. Among the married women, 91 percent lived with their husbands.

Only three percent of the clients were under the age of 20. Twenty-three percent were over the age of 35 . Fifty percent of the clients were between the age of 25-29. The mean and median ages of the clients were 27 and 28 respectively. Half of the clients were illiterate, 31 percent had completed primary education. Ninety-four percent of the clients were housewives.

The background characteristic of their husbands was also collected. Forty seven percent were illiterate, 18 percent completed the primary education, 25 percent had completed secondary, and nine percent studied secondary-level and above. Regarding occupation, 36 percent engaged in agricultural work, 33 percent were in business, 18 percent were in service and 13 percent engaged in transport sector (Table 5.1).

\section{REPORTED SYMPTOMS OF RTI/STD PROBLEMS}

Ninety-nine percent of the clients reported that the purpose of their visit was for abnormal vaginal discharge; 84 percent was for lower abdominal pain; 63 percent for pain in passing

\begin{tabular}{|lc|}
\hline \multicolumn{2}{|c|}{$\begin{array}{c}\text { Table 5.2 } \\
\text { Distribution of Clients by Reported RTI/STD Symptom }\end{array}$} \\
\hline Symptoms & Percent \\
\hline Abnormal vaginal discharge & 99.4 \\
Lower abdominal pain & 84.0 \\
Pain passing urine & 62.8 \\
Vaginal itching & 56.0 \\
Pain during coitus & 52.3 \\
Vaginal ulcer & 24.0 \\
Heavy bleeding & 15.4 \\
Genital ulcer & 10.8 \\
\hline $\mathrm{N}$ & 176 \\
\hline Multiple responses were possible
\end{tabular}
urine; 56 percent for vaginal itching and 52 percent pain during coitus. About one-fourth of the clients had come for vaginal ulcer, while about 11 percent for genital ulcer. Fifteen percent of the clients also reported that they came for the treatment of heavy bleeding (Table 5.2). The clients were asked where they usually went for treatment of such symptoms. Of the 176 cases, only 69 had previously sought treatment. Among the 69, 35 percent usually went to doctors, 23 percent 
FWV, 20 percent to a polly chikitsak (village doctor), 16 percent to traditional healers and seven percent to a homeopath practitioner.

\section{PREVALENCE OF REPRODUCTIVE HEALTH PROBLEMS}

After speculum examination, it was found that 98 percent of the clients had abnormal vaginal

\begin{tabular}{|c|c|}
\hline \multicolumn{2}{|c|}{$\begin{array}{c}\text { Table } 5.3 \\
\text { Percent Distribution of Clients by Indications of } \\
\text { Reproductive Health Problems }\end{array}$} \\
\hline Problems & Percent \\
\hline \multicolumn{2}{|c|}{ Lower abdomen pain } \\
\hline Yes & 66.5 \\
\hline No & 33.5 \\
\hline Total & 100.0 \\
\hline $\mathrm{N}$ & 161 \\
\hline \multicolumn{2}{|c|}{ Abnormal vaginal discharge } \\
\hline Vaginal & 82.9 \\
\hline Cervical & 14.8 \\
\hline None & 0.6 \\
\hline No information & 1.7 \\
\hline Total & 100.0 \\
\hline $\mathrm{N}$ & 176 \\
\hline \multicolumn{2}{|c|}{ Color of the discharge } \\
\hline Watery & 15.1 \\
\hline Curd like white & 65.7 \\
\hline Yellow & 15.1 \\
\hline Frothy & 1.7 \\
\hline No information & 2.4 \\
\hline Total & 100.0 \\
\hline $\mathrm{N}$ & 172 \\
\hline \multicolumn{2}{|c|}{ Amount of discharge } \\
\hline Scanty & 4.6 \\
\hline Moderate & 52.3 \\
\hline Profuse & 40.1 \\
\hline No information & 3.0 \\
\hline Total & 100.0 \\
\hline $\mathrm{N}$ & 172 \\
\hline \multicolumn{2}{|c|}{ Odor of discharge } \\
\hline Normal & 33.1 \\
\hline Foul smell & 61.0 \\
\hline No information & 5.9 \\
\hline Total & 100.0 . \\
\hline $\mathrm{N}$ & 172 \\
\hline \multicolumn{2}{|c|}{ Condition of cervix } \\
\hline Normal & 83.5 \\
\hline Abnormal & 11.4 \\
\hline No information & 5.1 \\
\hline Total & 100 \\
\hline $\mathrm{N}$ & 176 \\
\hline \multicolumn{2}{|l|}{ Abnormality } \\
\hline Reddish & 15.0 \\
\hline Erosion & 80.0 \\
\hline Old tear & 5.0 \\
\hline Total & 100.0 \\
\hline $\mathrm{N}$ & 20 \\
\hline
\end{tabular}
discharge: 83 percent had vaginal discharge and 15 percent had cervical discharge. No urethral discharge case was found. Discharge was curd-like in nearly two-thirds of the cases. Watery and yellow discharge made up 15 percent of the cases, while frothy discharge was found in only two percent of the cases. With regard to extent of discharge--more than half of the cases had moderate discharge; 40 percent were profuse and five percent had scanty discharge. The odor of the discharge was also diagnosed. Nearly two-thirds of the cases had foul smelling discharge, while another onethird had discharge without a noticeable smell. The providers also diagnosed the condition of the cervix and any kind of ulceration. It was found that the condition of the cervix was abnormal in 11 percent of the cases. Among the abnormalities 80 percent had unhealthy cervix (Table 5.3).

Two-thirds of the clients had lower abdominal pain. It was also found that lower abdominal pain increase with age. Of the 13 cases with symptoms of gonorrhoea and chamydia, nine had lower abdominal pain and six had cervicitis. Of the 13 cases with symptoms of trichomoniasis and bacterial vaginosis, seven had cervicitis and 11 had lower abdominal pain.

Current contraceptive use among the clients was also recorded. Nearly twothirds of the clients were using FP methods. Of them, 53 percent oral pill, 13 percent tubectomy and injection, 11 percent IUD, six percent condom; three percent of the clients' husband were vasetomized and only one client was practicing a traditional method. 


\section{CHAPTER 6}

\section{Lessons Learned and Policy Recommendations}

\section{LESSONS LEARNED}

- Service providers welcomed hands-on training

- Resource persons for hands-on training could be mobilized from within the existing service delivery system

- Timely and adequate supplies could be arranged from within the existing system

- A specific day of the week for RTI/STD services is practical

- Rearrangement of the service providers' room and client flow management ensured privacy

- Clients did not mind waiting for longer period for quality services

- More than one female service provider (FWV) at each H\&FWC is required for RTI/STD services

- Due to increased client flow, the program is suffering from a shortage of essential supplies

- Refresher training for the service providers is necessary

\section{POLICY RECOMMENDATIONS}

- Strengthening of RTI/STD services should be phased in, starting from the upgraded H\&FWCs

- Supply of essential materials, such as kerosene, cotton, gloves, bleaching powder and detergent should be increased

- Allotment and utilization of rooms at the H\&FWCs should be reconsidered (for example, OT could be used for examination)

- The provision of drugs for RTI/STDs should be revised to reflect the potential increase of clients

- MOs posted at the H\&FWCs should be present on the RTI/STD service delivery day to minimize the necessity for referral to higher levels

- On the day designated for RTI/STD services, a 'token system' for the management of client flow should be introduced

- FPCST team should be utilized as a training resource so that the service providers can remain at the site

- Service providers' training module should be revised, specially on the area of drug for RTI/STD management

- Providers should be oriented on the rational use of medicine

- RTI/STD services could be provided to men by SACMOs

- Comprehensive RTI/STD services can be strengthened in the FP-MCH service delivery system with the help of the following model:

\section{A Comprehensive RTI/STD Service Delivery Model}

- Assess the knowledge, attitude and practices of the service providers regarding RTI/STDs. Verify the facilities for RTI/STD services 
- Mobilize resource persons for hands-on training, and timely and an adequate logistics supply from within the existing system

- Design an on-site interventions according to the center's needs in a phased manner

- Orient the service providers on RTI/STD/HIV/AIDS and provide hands-on training for infection prevention

- Provide hands-on training on how to greet the client and document each client's profile; how to elicit history relevant to their major complaint and other related problems

- Reorganize the service providers' room (if needed) to ensure maximum privacy

- Serve the clients on a first-come-first-serve basis. Pregnant women, however should get priority over others clients

- Establish a referral mechanism for complicated cases and provide training on appropriate referral

- Assign one day each week for RTI/STD services

- Inform the community about the availability of services through FWAs

- Provide hands-on training for case management

- Prescribe necessary medicine for the client to buy from outside if appropriate drug is not available in the service facility (prescribe medicine for both the client and the partner)

- Provide hands-on training on how to tailor counseling to be relevant to diseases diagnosed and how to conduct follow-up visits

- Give a service provider at least three opportunities to render comprehensive RTI/STD services in the presence of the trainer

- Arrange a refresher training course after four months of initial training 


\section{REFERENCES}

Wasserheit, J., J.R. Harris, J. Chakraborty, B.A. Kay and K.J. Mason. 1987. "Reproductive Tract Infections in a Family Planning Population in Rural Bangladesh." Studies in Family Planning, Volume 20, No. 2, March/April 1989.

Chowdhury, S.N.M., Y.H. Ahmed, E. Karim and A.E. Masum. 1995. A Study to Determine the Prevalence of Reproductive Tract Infections Among Health Care Users of a Bangladesh Women's Health Coalition Clinic. BWHC, Bangladesh.

Hussain, M.A., G.S. Rahman, N.G. Banik and N. Begum. 1996. A Study of Prevalence of RTI/STDs in a Rural Area of Bangladesh. Save the Children, USA, Bangladesh.

Barkat, A., B. Khuda, J. Helali, A. Rahman, A.J. Faisel and M. L. Bose. 1994. Situation Analysis of Clinical Contraceptive Service Delivery System in Bangladesh. URC, Bangladesh.

Rahman, S., J.H. Chowdhury, M.S.H. Khan, T. Tariq and A.P.M.S. Rahman. 1996. MCH-FP Services in Rajshahi Division: A Situation Analysis. NIPORT, ACPR, and UNFPA, Bangladesh.

Islam, M.K., S.M. Hashemi and Z. Hossain. 1996. Situation Analysis of Quality of Care in Family Planning services. DRC, PDEU and World Bank, Bangladesh.

Chowdhury, S.N.M., I. Bhuiya, S.N. Huda and A.J. Faisel. 1997. Opportunities for Integration of RTI/STD Services into FP-MCH Programs. Population Council, Dhaka, Bangladesh.

MOHFW. 1986. Health and Family Welfare Center manual. Ministry of Health and Family Welfare, Dhaka, Bangladesh.

NIPORT. 1996. Training Manuals of Family Welfare Visitor. Dhaka, Bangladesh.

NIPORT. 1996. Training manuals of Sub Assistant Community Medical Officer. Dhaka, Bangladesh. 


\section{Appendix 1}

STRENGTHENING RTI/STD SERVICES IN H\&FWCS

\section{Checklist for the Observation of the Service delivery Process}

1. Name of H\&FWCs: Elenga 1

Narandia 2

Sahadevpur 3

Paikara 4

2. Thana: Khalihati

4. District: Tangail

5. Date of Observation:

6. Name of the observer

7. Did the service provider greet you?

$\begin{array}{ll}\text { Yes } & 1 \\ \text { No } & 2\end{array}$

8. What problems did you stated to the service provider?

\begin{tabular}{|l|c|c|}
\hline \multicolumn{1}{|c|}{ Problems } & Yes & No \\
\hline A. Unusual vaginal discharge & 1 & 2 \\
\hline B. Lower abdominal pain & 1 & 2 \\
\hline C. Genital ulcer & 1 & 2 \\
\hline D. Genital itching & 1 & 2 \\
\hline E. Vaginal itching & 1 & 2 \\
\hline F. Heavy bleeding & 1 & 2 \\
\hline G. Burning sensation during micturation & 1 & 2 \\
\hline H. Painful coitus & 1 & 2 \\
\hline I. Warts in genitalia & 1 & 2 \\
\hline
\end{tabular}

9. Who took the history in relation to disease?

\begin{tabular}{|l|c|c|}
\cline { 2 - 3 } \multicolumn{1}{c|}{} & Yes & No \\
\hline A. SACMO & 1 & 2 \\
\hline B. FWV & 1 & 2 \\
\hline C. FWA & 1 & 2 \\
\hline D. Aya & 1 & 2 \\
\hline
\end{tabular}


10. What information did the service provider take and was it recorded and if so where?

\begin{tabular}{|c|c|c|c|c|c|}
\hline \multirow[t]{2}{*}{ Information } & \multicolumn{2}{|c|}{ Took } & \multicolumn{2}{|c|}{ Recorded } & \multirow[t]{2}{*}{ Where } \\
\hline & Yes & No & Yes & No & \\
\hline A. Name & & & & & \\
\hline B. Age & & & & & \\
\hline C. Address & & & & & \\
\hline D. Occupation of the client & & & & & \\
\hline E. Occupation of the spouse & & & & & \\
\hline F. Last menstrual period & & & & & \\
\hline G. Length of menstrual cycle & & & & & \\
\hline H. Amount of bleeding & & & & & \\
\hline I. Who did the last delivery & & & & & \\
\hline J. Place of last delivery & & & & & \\
\hline K. History of abortions/MR/D\&C & & & & & \\
\hline L. Unusual vaginal discharge & & & & & \\
\hline M. Lower abdominal pain & & & & & \\
\hline N. Ulcer in genitalia & & & & & \\
\hline O. Itching in genitalia & & & & & \\
\hline P. Itching in vagina & & & & & \\
\hline Q. Burning sensation during micturation & & & & & \\
\hline R. Painful coitus & & & & & \\
\hline S. History of FP method use & & & & & \\
\hline T. History of dysentery & & & & & \\
\hline U. History of diabetes & & & & & \\
\hline
\end{tabular}

11. Did the provider perform pelvic examination?

Yes 1

11_1. If yes, whether any instruments was used?

Yes 1

No 2

11_2. If yes, how did she decontaminate the examination table before the pelvic examination? (Tick what you observe. Multiple answer is possible)

\begin{tabular}{|l|c|c|}
\hline \multicolumn{1}{|c|}{ Way of decontamination } & Yes & No \\
\hline A. Wipe with chlorine solution & 1 & 2 \\
\hline B. Wipe with wet cloth & 1 & 2 \\
\hline C. Wipe with soap water & 1 & 2 \\
\hline D. Wipe with savlon & 1 & 2 \\
\hline E. Changed sheet & 1 & 2 \\
\hline
\end{tabular}


12. What did the service provider do after pelvic examination?

\begin{tabular}{|l|c|c|}
\hline \multicolumn{1}{|c|}{ Management of RTI/STD } & Yes & No \\
\hline A. Told the clients about the diagnosis & 1 & 2 \\
\hline B. Medicine given for client & 1 & 2 \\
\hline C. Medicine given for partner & 1 & 2 \\
\hline D. Prescribe medicine for partner & 1 & 2 \\
\hline E. Referred & 1 & 2 \\
\hline F. Counseled & 1 & 2 \\
\hline
\end{tabular}

12_1. If treatment was given, what type of medicine the provider gave for the following symptoms?

\begin{tabular}{|c|c|}
\hline & Treatment \\
\hline & \begin{tabular}{|l|} 
Metronidazole------------1 \\
\end{tabular} \\
\hline & Antibiotic-----------------2 \\
\hline & Anti fungal----------------3 \\
\hline & Anti spasmodic----------4 \\
\hline & Analgesic------------------5 \\
\hline & Oral pill---------------------6 \\
\hline & Condom--------------------7 \\
\hline Symptoms & \\
\hline A. Unusual vaginal discharge & \\
\hline B. Lower abdominal pain & \\
\hline C. Genital ulcer & \\
\hline D. Genital itching & \\
\hline E. Vaginal itching & \\
\hline F. Heavy bleeding & \\
\hline G. Burning sensation during micturation & \\
\hline H. Painful coitus & \\
\hline I. Warts in genitalia & \\
\hline
\end{tabular}

12_2. If counseling was done, what were the elements of counseling?

\begin{tabular}{|l|c|c|}
\hline \multicolumn{1}{|c|}{ Elements of counseling } & Yes & No \\
\hline A. Informed the client about good personal hygiene & 1 & 2 \\
\hline B. Informed the client about the doses of the medicine & 1 & 2 \\
\hline C. Emphasis given on condom use/safe sex & 1 & 2 \\
\hline D. Emphasis given on partner's treatment & 1 & 2 \\
\hline E. Provided Information on follow-up & 1 & 2 \\
\hline
\end{tabular}




\section{Appendix 2}

\section{STRENGTHENING RTI/STD SERVICES IN H\&FWCS \\ Population Council, Dhaka \\ [ SACMO and FWV]}

ID No:

১। आপनाর নাম:

२। পদदी:

SACMO 1

FWV

2

৩। যে ইউনিয়ন পরিবার কল্যাণ কেন্দ্রে কাজ করেন, দয়া করে সেই সেবা কেন্দ্রে টিক চিহু দিন।

$\begin{array}{ll}\text { এলেঙ্গা } & 1 \\ \text { নারানদিয়া } & 2\end{array}$

8। কত বছর আগে আপনি মৌলিক প্রশিক্ষণ গ্রহণ করেছেন?

Q1 'প্রজনनতন্ত্রের সংক্রমণ' (Reproductive Tract Infection- RTI) কি ?

৬। কমপক্ষ 8টি সাধারন 'প্রজনनতট্রের সংক্রমণের' (RTI) নাম উল্rেখ করুন। কি কি কারণে প্রজননতত্রের সংক্রমণ হয় বলে আপনি মনে করেন? প্রতিটি সংক্রামনের জন্য কমপছক্ষ ২ টি কারন উল্লেখ করুন ।

\begin{tabular}{|c|c|}
\hline প্রজননতত্র্রের সংক্রমণের নাম & কি কার্রণে হয় \\
\hline A. & $\begin{array}{l}\mathrm{A} 1 \\
\mathrm{~A} 2\end{array}$ \\
\hline B. & $\begin{array}{l}\mathrm{B} 1 . \\
\mathrm{B} 2 .\end{array}$ \\
\hline C. & $\begin{array}{l}\mathrm{C} 1 . \\
\mathrm{C} 2 . \\
\end{array}$ \\
\hline D. & $\begin{array}{l}\text { D1. } \\
\text { D2. }\end{array}$ \\
\hline
\end{tabular}

१। यৌন বাহিত রোগ (Sexually Transmitted Disease - STD) কি?

b। কমপক্ষে ৪টি সাধারন যৌন বাহিত রোগের (Sexually Transmitted Disease) নাম উন্দেখ করুন?
A
B
C
D

৯। প্রজনनতন্ত্রের সংক্রমনের চিকিৎসা यদি না করা হয় তা হনে ফলাফল কি হতে পারে?

(কমপক্ষে ৩টি ফলাফল উন্মে করুন)

A

B

C

১০। যৌন বাহিত রোগের চিকিৎসা যদি না করা হয় তা হলে ফলাফল কি হতে পারে,

(কমপক্ষে ৩টি ফলাফল উল্দেখ করুন)
A
B
C 
এইচ,আই,ভি,এইডস (HIV/AIDS) এর নাম खনেছেন?

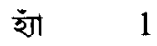

ना 2

১২। কি কারনে এইচ,আই,ভি,/এইডস হয়? (কমপক্ষ ৪টি কারন উন্দেখ করুন)

A

B

C

D

১৩। কিভাবে এইচ,আই,ভি,/এইডস এর প্রতিরোধ করা যায়? (কমপক্ষে ৪টি প্রতিরোধ ব্যবস্থা উন্দেখ করুন)

A

B

C

D

১৪। এইচ,আই,ভি,/এইডস এর চিকিৎসা কি?

se 1

আপনার সেবা গ্রহীতাদের মধ্যে নীচের উপসর্গঞলোর মধ্যে কোন কোন ওনো সচরাচর পরিলক্ষিত হয়? এবং কোন বয়সের মহিলা/পুরুষদের মধ্যে পরিলক্ষিত হয়? দয়া করে টিক চিহ্ দিন।

\begin{tabular}{|c|c|c|c|c|c|c|}
\hline \multirow{3}{*}{ উপসর্গ } & \multirow{2}{*}{\multicolumn{2}{|c|}{ কোন কোন উপসর্গ }} & \multicolumn{4}{|c|}{ কোন বয়সের } \\
\hline & & & \multicolumn{2}{|c|}{ ২০ বৎসরের নীচে } & \multicolumn{2}{|c|}{ ২০ বৎসরের উর্দ্ধে } \\
\hline & शों & ना & शों & ना & शों & ना \\
\hline \multicolumn{7}{|l|}{ মহিলাদের মব্য } \\
\hline A. সাদাস্রাব/ধাতু যায় & 1 & 2 & 1 & 2 & 1 & 2 \\
\hline B. তলপপেটে ব্যাথা & 1 & 2 & 1 & 2 & 1 & 2 \\
\hline C. মাসিকের রাস্তায় ঘা & 1 & 2 & 1 & 2 & 1 & 2 \\
\hline D. মাসিকের রাস্তায় হুলকানী & 1 & 2 & 1 & 2 & 1 & 2 \\
\hline E. প্রস্রাবে জ্বালাপোড়া & 1 & 2 & 1 & 2 & 1 & 2 \\
\hline F. যৌনাঙ্গে घा & 1 & 2 & 1 & 2 & 1 & 2 \\
\hline G. সহবাসে ব্যাথা & 1 & 2 & 1 & 2 & 1 & 2 \\
\hline \multicolumn{7}{|l|}{ भूख़्टखज मढষ্য } \\
\hline H. পৃরুযের নিঙ্গ থেকে পুঁজ & 1 & 2 & 1 & 2 & 1 & 2 \\
\hline I. ধাতু যাওয়া & 1 & 2 & 1 & 2 & 1 & 2 \\
\hline J. অন্ডকোমে ব্যাথ & 1 & 2 & 1 & 2 & 1 & 2 \\
\hline K. উরুতত বাগী & 1 & 2 & 1 & 2 & 1 & 2 \\
\hline L. পুরুষাঙ্গে ঘা & 1 & 2 & 1 & 2 & 1 & 2 \\
\hline M. পুরুষাত্সে হূলকানী & 1 & 2 & 1 & 2 & 1 & 2 \\
\hline N. প্রস্রাবে জ্বালাপোড়া & 1 & 2 & 1 & 2 & 1 & 2 \\
\hline
\end{tabular}

১৬। এইসব উপসর্গগুনো নিয়ে যারা আপনার কাছে আসেন - তাদের জন্য আপনি কি করে থাকেন?

\begin{tabular}{|l|c|c|}
\hline & श्रा & ना \\
\hline A. চিকিৎসা দিই & 1 & 2 \\
\hline B. পরামর্শ দিই & 1 & 2 \\
\hline
\end{tabular}

১৬-১। यদি চিকিৎসা/পরামর্শ দেন, তবে কোন কোন উপসর্গের জন্য কি কি চিকিৎসা/পরামর্শ দেন-দয়া করে লিখুন।

\begin{tabular}{|c|c|c|}
\hline উপসर्भ & कि চिकिৎना দেन & कি পর্রামর্শ দেন \\
\hline A. সাদা স্রাব/ধাতু যায় & $\begin{array}{l}\mathrm{A} 1 \_1 . \\
\mathrm{A} 122 . \\
\mathrm{A} 13 .\end{array}$ & $\begin{array}{l}\mathrm{A} 2 \_1 \\
\mathrm{~A} 2 \_2 . \\
\mathrm{A} 23\end{array}$ \\
\hline B. তলপপটে ব্যাথা & $\begin{array}{l}\mathrm{B} 1 \_1 . \\
\mathrm{B} 1-2 . \\
\mathrm{B} 13 .\end{array}$ & $\begin{array}{l}\mathrm{B} 2 \_1 . \\
\mathrm{B} 2-2 . \\
\mathrm{B} 23\end{array}$ \\
\hline C. যাসিকের রাস্তায় ঘা & $\begin{array}{l}\mathrm{C} 1{ }_{-} . \\
\mathrm{C} 12\end{array}$ & $\begin{array}{l}\mathrm{C}^{2}-1 . \\
\mathrm{C}_{2}-2 .\end{array}$ \\
\hline
\end{tabular}




\begin{tabular}{|c|c|c|}
\hline উপनর্গ & कि চিকিeসा দেন & কি পরামর্শ দেন \\
\hline & C1 3. & $\mathrm{C} 23$. \\
\hline D. মাসিকের রাস্তায় হুলকানি & $\begin{array}{l}\text { D1_1. } \\
\text { D1_2. } \\
\text { D1-3. }\end{array}$ & $\begin{array}{l}\text { D2_1. } \\
\text { D2_2. } \\
\text { D2 } 3\end{array}$ \\
\hline E. প্রস্রাবে জ্বালাপোড়া & $\begin{array}{l}\text { E1_1. } \\
\text { E1_2. } \\
\text { E1_3. }\end{array}$ & $\begin{array}{l}\text { E2_1. } \\
\text { E2_2. } \\
\text { E2 } 3 .\end{array}$ \\
\hline F. বৌনাঞ্সে घा & $\begin{array}{l}\text { F1_1. } \\
\text { F1_2. } \\
\text { F13. }\end{array}$ & $\begin{array}{l}\mathrm{F} 2-1 \\
\mathrm{~F} 2-2 \\
\mathrm{~F} 2\end{array}$ \\
\hline G. সহবাসে ব্যথা & $\begin{array}{l}\text { G1_1. } \\
\text { G1_2. } \\
\text { G1 } 3 .\end{array}$ & $\begin{array}{l}\mathrm{G} 2 \_1 . \\
\mathrm{G} 2{ }^{-} 2 . \\
\mathrm{G} 23 .\end{array}$ \\
\hline H. পুরুমের লিন্স থেকে পুঁজ & $\begin{array}{l}\mathrm{H} 1 \_1 . \\
\mathrm{H} 1 \_2 . \\
\mathrm{H} 13 .\end{array}$ & $\begin{array}{l}\mathrm{H} 2-1 . \\
\mathrm{H} 2-2 . \\
\mathrm{H} 2-3 .\end{array}$ \\
\hline I. ধাতু যাওয়া & $\begin{array}{l}\text { I1_1. } \\
\text { I1_2. } \\
\text { I1_ } 3 .\end{array}$ & $\begin{array}{l}\mathrm{I} 2-1 . \\
\mathrm{I}_{2}-2 . \\
\mathrm{I} 23 .\end{array}$ \\
\hline J. অন্ডকোযে ব্যথা & $\begin{array}{l}\mathrm{J} 11_{1} 1 . \\
\mathrm{J} 1 \text { 2. } \\
\mathrm{J} 1{ }^{2} 3 .\end{array}$ & $\begin{array}{l}\mathrm{J} 2 \_1 \\
\mathrm{~J} 2-2 \\
\mathrm{~J} 23\end{array}$ \\
\hline K. উরুতত বাগী & $\begin{array}{l}\mathrm{K} 1 \_1 . \\
\mathrm{K} 1-2 . \\
\mathrm{K} 13 .\end{array}$ & $\begin{array}{l}\mathrm{K} 2-1 . \\
\mathrm{K} 2-2 . \\
\mathrm{K} 23 .\end{array}$ \\
\hline L. পুরুষান্গে ঘা & $\begin{array}{l}\text { L1_1. } \\
\text { L1_2. } \\
\text { L1 } 3 .\end{array}$ & $\begin{array}{l}\mathrm{L} 2 \_1 . \\
\mathrm{L} 2-2 . \\
\mathrm{L} 23 .\end{array}$ \\
\hline M. পুরুষান্সে দূলকানি & $\begin{array}{l}\text { M1_1. } \\
\text { M1_2. } \\
\text { M1 } 3 .\end{array}$ & $\begin{array}{l}\text { M2_1. } \\
\text { M2_2. } \\
\text { M2 } 3 .\end{array}$ \\
\hline
\end{tabular}

১৬-2। প্রজননতত্ত্রে সংত্রমিত বা যৌন বাহিত রোগীদের যৌনাঙ্গ পরীর্ষ করেন?

$$
\begin{array}{ll}
\text { एँा } & 1 \\
\text { ना } & 2
\end{array}
$$

১৬-৩। আপনি কি প্রজননতত্তের সংক্রমণের বা লৌৗন বাহিত রোগের সেবা গ্রহীতার স্বামী ও স্ত্রীর চিকিৎসার ব্যবস্থা একসাথে করে থাকেন?

$$
\begin{array}{ll}
\text { इा } & 1 \\
\text { ना } & 2
\end{array}
$$

\begin{tabular}{|c|c|c|}
\hline & शाँ & ना \\
\hline A. ত্ত্রীকে ঔষধ দিই & 1 & 2 \\
\hline B. স্বামীবক ঔষধ দিই & 1 & 2 \\
\hline C. স্ত্রীকে ব্যবস্থাপত্র দিই & 1 & 2 \\
\hline D. স্যামীকে ব্যবস্থাপত্র দিই & 1 & 2 \\
\hline E. রেফার করি & 1 & 2 \\
\hline F. স্ত্রীকে পরামর্শ দিই & 1 & 2 \\
\hline G. স্বামীকে পরামর্শ দিই & 1 & 2 \\
\hline
\end{tabular}

১৬-8। यদি স্বামী ও স্ত্রীর চিকিৎসার ব্যবস্থা একসাথে করে থাকেন - তবে নিচের কোন কোন পদ্ধতিখ্যনো অনুসরন করেন?

১৭। প্রজননতন্ত্রের সংক্রমন বা লৌৗন বাহিত রোগের (RTI/STD) চিকিৎসার জন্য কি কি ঔ্যষধ আপনার সেবা কেট্ত্রে পাওয়া যায় - তা কি

\begin{tabular}{|c|c|c|}
\hline \multirow[t]{2}{*}{ ঔষধ } & \multicolumn{2}{|c|}{ প্রয়োজনানুসারে সরবরাহ } \\
\hline & श्या & ना \\
\hline A. মেট্রোনিডাজল 800 মি. গাম & 1 & 2 \\
\hline B. মেট্টোনিডাজল ২৫০ মি. গ্রাম & 1 & 2 \\
\hline C. জেনশন ভায়লেট & 1 & 2 \\
\hline
\end{tabular}
প্রত্যোজনানুসারে সরবরাহ পাওয়া যায়? 


\begin{tabular}{|c|c|c|}
\hline D. ডক্সিসাইক্লিন ১০০ Aি. গ্রাম & 1 & 2 \\
\hline E. টেট্রাসাইক্সিন ৫০০ মি. গাম & 1 & 2 \\
\hline F. ইরিথরোমাইসিন ৫০০ মি. গ্রাম & 1 & 2 \\
\hline G. निষ्ठाটিन & 1 & 2 \\
\hline H. সিত্রোফ্যক্সাসিন ৫০০ মি. গ্রাম & 1 & 2 \\
\hline I. কেনামাইসিন & 1 & 2 \\
\hline
\end{tabular}

so 1

দিনে গড় পরতা মোট কতজন রোগী দেখেন? এবং তার মধ্যে কতজন প্রজননতত্রের সংক্রমণের বা শ্যীনবাহিত রোগের রোগী?
A. রোট রোগী
B. প্রজনनতন্তের/:যৌনবাহিত সংক্রমণের রোগী

১৯।

\begin{tabular}{|c|c|c|c|}
\hline যন্ত্রপাতির নাম & इ্যা & ना & मश्थ्या \\
\hline A. সिহস - Sim's vaginal Speculum & 1 & 2 & \\
\hline B. কাসকোস - Cuscos Vaginal Speculum & 1 & 2 & \\
\hline C. স্পঞ্জান্ডিং ফরসেপ - Sponge Holding Forcep & 1 & 2 & \\
\hline D. গালী পট - Gully pot & 1 & 2 & \\
\hline E. ইन्भदমেন্ট बট্ব - Instrument tray & 1 & 2 & \\
\hline F. লিফটার - Lifter & 1 & 2 & \\
\hline G. भाष्म - Gloves & 1 & 2 & \\
\hline H. Spot light & 1 & 2 & \\
\hline J. Cotton ball & 1 & 2 & \\
\hline
\end{tabular}

২০। আপনার সেবা কেন্দ্রে কি গাভস নিয়মিতভাবে সরবরাহ পাওয়া যায়?

$$
\begin{array}{ll}
\text { इाँ } & 1 \\
\text { ना } & 2
\end{array}
$$

২১। आপনার সেবা কেন্দ্রে MR করা হয়?

$\begin{array}{ll}\text { श्या } & 1 \\ \text { ना } & 2\end{array}$

২২। MR করা হলে কিভাবে MR Cannula জীবাণুমুক্ত করেন?

A

B

C

২৩। MR এর পরে সেবা গ্রহীতার Infection না হওয়ার জন্য সচারচর আপনি কি করর থাকেন?
A. Full course ঔ্বৈধ দিই
B. আংশিক ঔষষ দিই
C. ব্যবস্থাপত্র দিই

২৪। ঔষধ দিয়ে থাকলে কি ধরনের ঔষধ দেন?

A

B

$\mathrm{C}$

\begin{tabular}{|c|c|c|}
\hline यন্ত্রপাতি & श्या & ना \\
\hline A. অটোর্রেণ & 1 & 2 \\
\hline B. যন্ত্রপাতি সিদ্ধ করার পাত্র (Sterilizer) & 1 & 2 \\
\hline C. চুলা & 1 & 2 \\
\hline D. বালতি & 1 & 2 \\
\hline E. ব্রাস (পুরাতন টূথ ব্রাসও হতে পারে) & 1 & 2 \\
\hline F. शাকनि (Strainer) & 1 & 2 \\
\hline G. নাড়ার কাঠি & 1 & 2 \\
\hline H. ন্লিচিং পাউডার মাপার পাত্র & 1 & 2 \\
\hline
\end{tabular}

২৫। জীবানু মুক্ত করনের জন্য আপনার সেবা কেন্দ্রে কোন কোন ধরতের ব্যবহার ঊপযোগী যন্ত্রপাতি রয়েছে? 
২৬। নীচের কোন কোন সামগ্রী সংক্রমণ (Infection) প্রতিরোধের জন্য আপনার সেবা কেন্দ্রে সরবরাহ পাওয়া যায়?

\begin{tabular}{|c|c|c|}
\hline সামগ্রী & इ্যা & ना \\
\hline A. ब্রিচিং পাউডার & 1 & 2 \\
\hline B. শুড়ো সাবান & 1 & 2 \\
\hline C. বার সাবান & 1 & 2 \\
\hline D. ডেটল/স্যাভলন & 1 & 2 \\
\hline E. আয়োডিন & 1 & 2 \\
\hline F. স্পিরিট & 1 & 2 \\
\hline G. ক্রোরোহেক্সিডিন/হিবিসল & 1 & 2 \\
\hline H. श्राडস & 1 & 2 \\
\hline
\end{tabular}

২१। আপনার সেবাকেন্দ্রে যন্ত্রপাতি কে পব্রিক্ষার করেন?
A. आয়া
B. FWV
C. SACMO

$\begin{array}{ll}\text { श्या } & \text { ना } \\ 1 & 2 \\ 1 & 2 \\ 1 & 2\end{array}$

২৮। আপনার সেবাকেন্দ্রে যন্ত্রপাতি কে বিশ্যেধধন করেন ?
A. आয়া
B. FWV
C. SACMO

$\begin{array}{ll}\text { शँ } & \text { ना } \\ 1 & 2 \\ 1 & 2 \\ 1 & 2\end{array}$

২৯। আপনার লেবা কেন্দ্রে সেবাদানের জন্য যন্ত্রপাতি চুড়ান্ত ভাবে কে টেবিলে সাজান?

$\begin{array}{lll} & \text { श्या } & \text { ना } \\ \text { A. आয়া } & 1 & 2 \\ \text { B. FWV } & 1 & 2 \\ \text { C. SACMO } & 1 & 2\end{array}$

৩০ 1 আপনার সেবা কেন্ট্রে এইসব সংক্রমণের সেবার মান উন্নয়নের লক্ষে আপনার কি বোন পরামর্শ আছে? দয়া কর্রে উল্পেখ করুন।

A

B

C 


\section{Appendix 3}

\section{STRENGTHENING RTI/STD SERVICES IN H\&FWCS}

Population Council, Dhaka

[FPI and FWA]

ID No:

১। आপনার नाম:

२। পদবी: FPI 1, FWA 2

৩। যে ইউনিয়ন পরিবার কল্যাণ কেন্দ্রে কাজ করেন, দয়া করে সেই সেবা কেন্দ্রের টিক চিহৃ দিন।

$\begin{array}{ll}\text { এলেঙা } & 1 \\ \text { নারানদিয়া } & 2 \\ \text { সাহদেবপুর } & 3 \\ \text { পাইকরা } & 4\end{array}$

81 আমাদের দেলে পুরুষ ও মহিলাদের মধ্যে সাদাস্রাব/ধাতু যাওয়া, যৌনাঙ্গে ঘা বা চূলকানি ইত্যাদি বিভিন্ন ধরনের প্রজননতন্ত্রের সংক্রামন / যৌন বাহিত জনিত সমস্যা (Reproductive Tract Infection _ RTI / Sexually Transmitted Disease _ STD) দেখা याয়। আপনি কি আপনার এনাকার জনসাধারণের মধ্য এই ধরনের সমস্যার কথা ওনেছেন?

$\begin{array}{ll}\text { शंग } & 1 \\ \text { ना (প्रশ্ন 8-১ প্রযোজ্য नয়) } & 2\end{array}$

8-১। यদি এই ধরনের সমস্যার কথা ওনে থাকেন তবে নীচের সমস্যা গুনোর মখ্যে কোন কোনটির কথা ওনেছেন এবং কোন বয়সের মহিলা/পুরুষদের মধ্যে আছে বলে তেছেন? দয়া করে টিক চিহ্ন দিন।

\begin{tabular}{|c|c|c|c|c|c|c|}
\hline \multirow[b]{2}{*}{ উপসর্গ/সयসगा } & \multicolumn{2}{|c|}{ কোন কোন সমস্যা } & \multicolumn{4}{|c|}{ কোন বয়সের } \\
\hline & श्या & ना & \multicolumn{2}{|c|}{$\begin{array}{l}\text { ২০ বৎসরের নীচে } \\
\text { । হা =1, না=2) }\end{array}$} & \multicolumn{2}{|c|}{$\begin{array}{l}\text { ২০ বৎসরের উর্ধ } \\
\text { (হ্যা =1, না=2) }\end{array}$} \\
\hline \multicolumn{7}{|l|}{ মरिলাদের্ন মব্য } \\
\hline A. সাদাস্রাব/ধাতু যায় & 1 & 2 & i & 2 & 1 & 2 \\
\hline B. তনপেটে ব্যাথা & 1 & 2 & 1 & 2 & 1 & 2 \\
\hline C. মাসিকের রাস্তায় ঘা & 1 & 2 & 1 & 2 & 1 & 2 \\
\hline D. মাসিকের রাষ্তায় চুলকানী & 1 & 2 & 1 & 2 & 1 & 2 \\
\hline E. প্রস্রাবে জ্বালাপোড়া & 1 & 2 & 1 & 2 & 1 & 2 \\
\hline F. যৌনাঙ্গে ঘা & 1 & 2 & 1 & 2 & 1 & 2 \\
\hline G. সহবাসে ব্যাথা & 1 & 2 & 1 & 2 & 1 & 2 \\
\hline \multicolumn{7}{|l|}{ পুর্য়শর মণধ্য } \\
\hline H. পুরুষের লিঙ থেকে পুঁজ & 1 & 2 & 1 & 2 & 1 & 2 \\
\hline 1. ধাতু যাওয়া & 1 & 2 & 1 & 2 & 1 & 2 \\
\hline J. অন্ডকোষে ব্যাথা & 1 & 2 & 1 & 2 & 1 & 2 \\
\hline K. উরুতে বাগী & 1 & 2 & 1 & 2 & 1 & 2 \\
\hline L. পুরুষাঙ্গ ঘা & 1 & 2 & 1 & 2 & 1 & 2 \\
\hline M. পুরুষাঙ্গে চলকানী & 1 & 2 & 1 & 2 & 1 & 2 \\
\hline N. প্রস্রাবে জ্বালাপোড়া & 1 & 2 & 1 & 2 & 1 & 2 \\
\hline
\end{tabular}


এই ষরনের সমস্যাখলো কি কারনে হয়? এবং কিভাবে এর প্রত্রোধ করা যায় বলে আপনি মনে করেন?

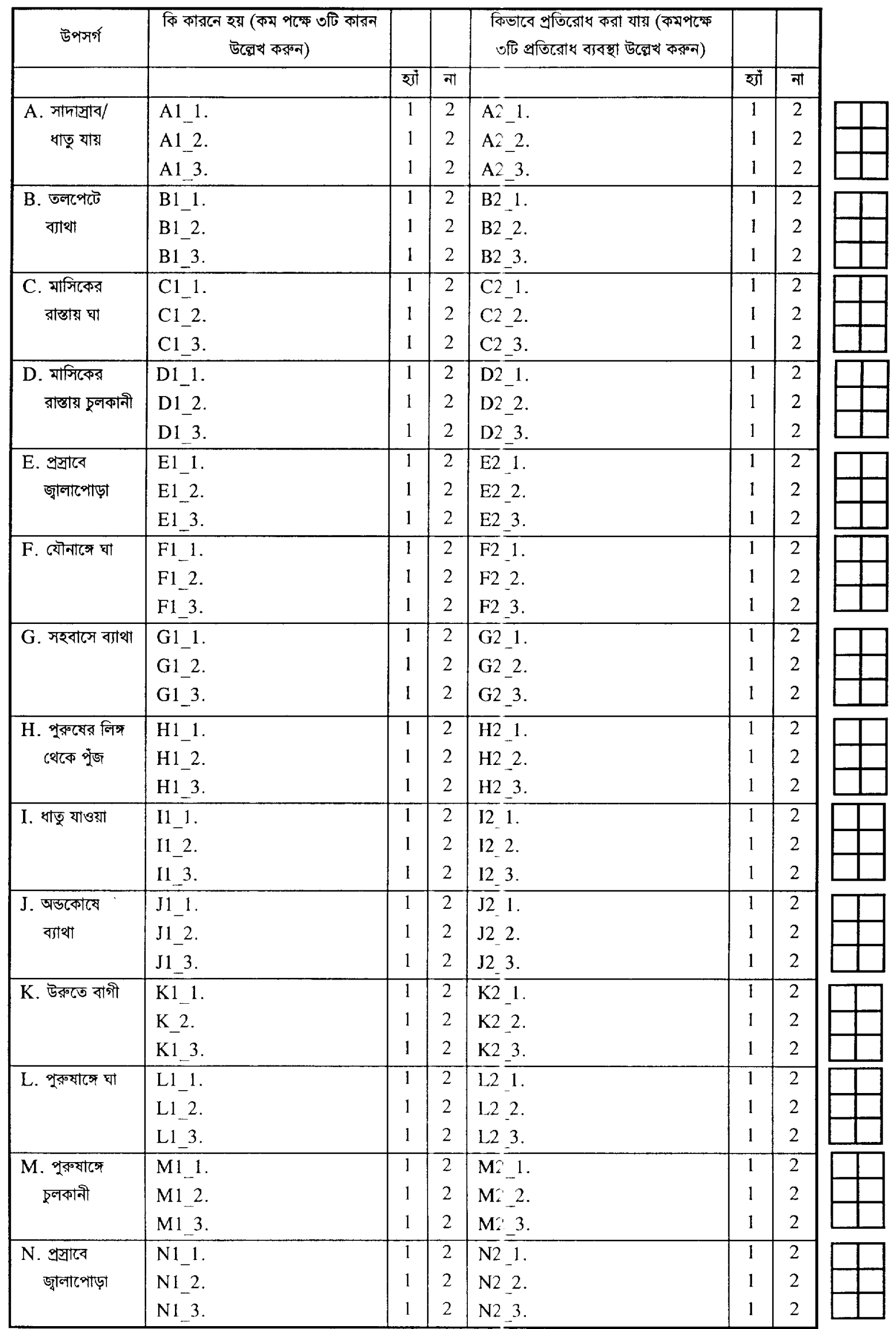


আপনার কাছ মহিলা/পুরুষরা কি কখনো নীচের সমস্যাওলোর জন্য পরামর্শ চেয়ে পাকেন? পরামর্শ দিয়ে থাকলে নিচের কোন কোন সমস্যার জন্য কি কি পরামর্শ দেন?

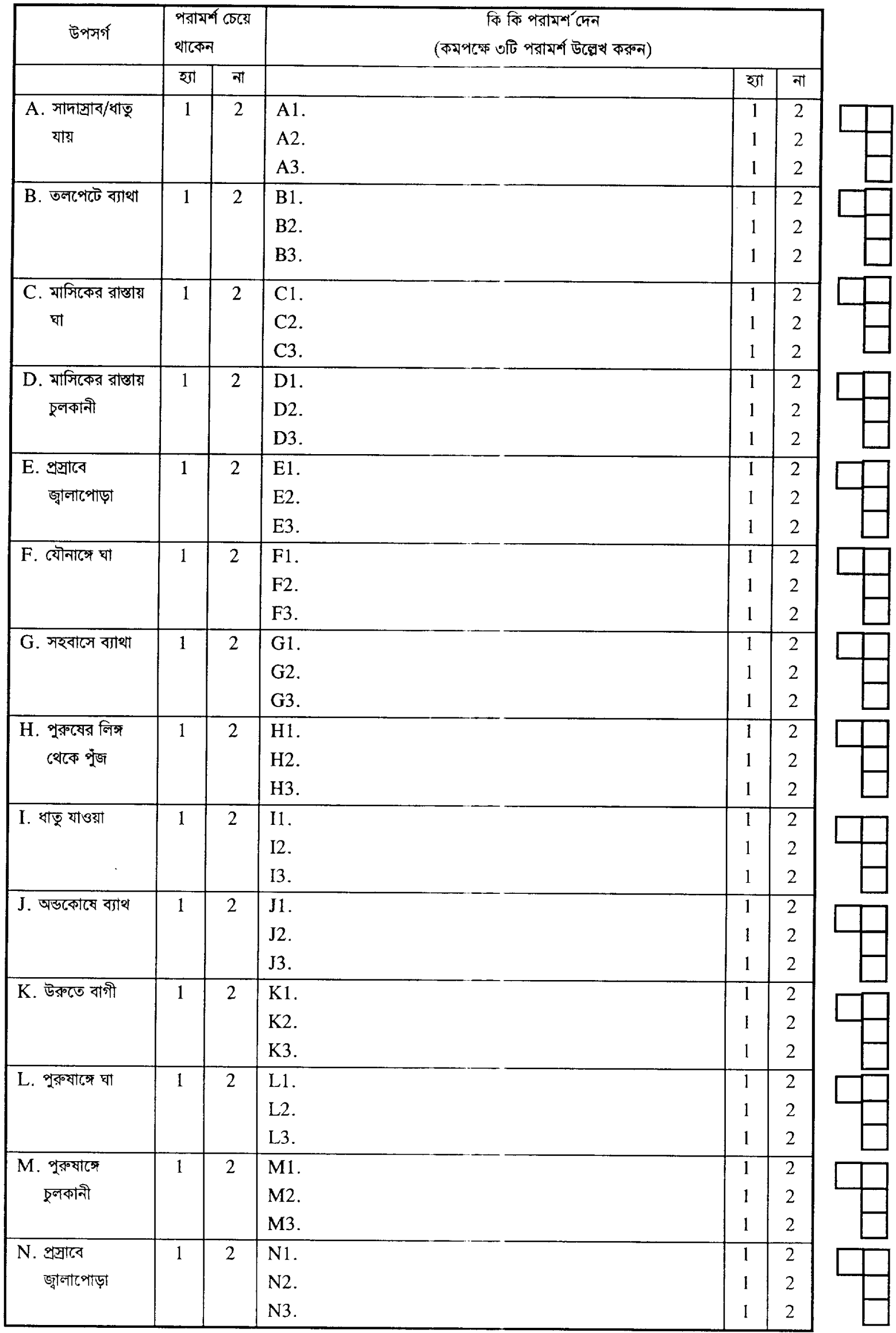


(৩টি মন্তব্য লिপিবদ্ধ করুন)

\begin{tabular}{|l|l|l|}
\hline & शां & ना \\
\cline { 2 - 4 } B. & 1 & 2 \\
C. & 1 & 2 \\
\hline
\end{tabular}

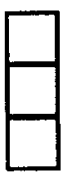

৮। এইচ, আই, ভি/এইডস (HIV/AIDS) এর কথা ওনেছেন?

$\begin{array}{ll}\text { शंगा } & 1 \\ \text { ना } & 2\end{array}$

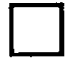

৯। कি কারনে এইডস হয় বনে মনে করেন? (কমপক্ষে ৩টি কারন উল্পেখ করুন)

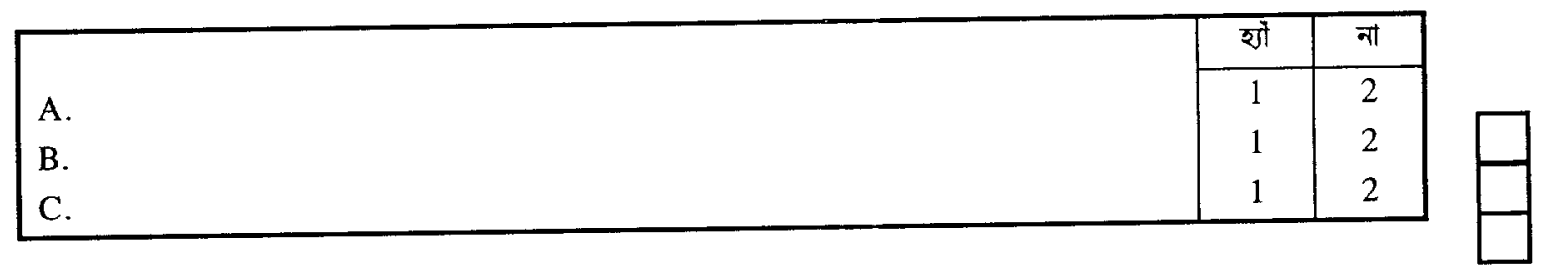

১০। কিভাবে এইডস এর প্রতিরোধ করা যায়? (কমপক্ষে ৩টি প্রতিরোধ ব্যবস্থা উদ্ধে করুন)

\begin{tabular}{|c|c|c|}
\hline & ख्याँ & না \\
\hline A. & 1 & 2 \\
\hline B. & 1 & 2 \\
\hline C. & 1 & 2 \\
\hline
\end{tabular}




\section{Appendix 4}

\section{STRENGTHENING RTI/STD SERVICES IN H\&FWCs}

Population Council, Dhaka

\section{সেবাকেন্দ্রে সাধারন সুযোগসুবিধা নিরীক্ষা সৃচী}

১। কেন্দ্রে কর্মরত কর্মী সংখ্যা কত? (গত মাসে কর্মরত কর্মীদের সংখ্যা লিখুন)

\begin{tabular}{|c|c|}
\hline कर्यो & কর্মীর সংখ্যা \\
\hline মেডিক্যাল অফিসার & \\
\hline সাকমো & \\
\hline এফ ডব্লিউ ভি & \\
\hline কারমাসিস্ট & \\
\hline আয়া & \\
\hline নৈশ প্রহরী & \\
\hline
\end{tabular}

২। পরিবার পরিকল্পনা ও মা ও শিফ্য স্বাস্থ্য বিষয়ক কোন কোন সেবা এই :কন্দ্রে দেওয়া হয়ে থাকে?

\begin{tabular}{|c|c|}
\hline সেবার নাম: & खाँ \\
\hline কনডম & 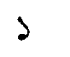 \\
\hline খাবার বড়ি & 2 \\
\hline ইনজেকশন & $د$ \\
\hline আই, ইউ, ডি & $s$ \\
\hline নরপ্পান্ট & 2 \\
\hline লাইগগেশন & $>$ \\
\hline ভ্যাসেকটমী & 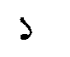 \\
\hline মাসিক নিয়মিত করন (এম, আর) & 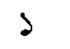 \\
\hline গর্ভবতী মায়ের যত্ম & 2 \\
\hline প্রসোবত্তোর যত্ন & 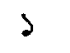 \\
\hline সাধারন প্রজনন স্বাস্থ্য সেবা & \\
\hline (general reproductive health) & 2 \\
\hline
\end{tabular}

৩। এই ক্লিনিকে সেবা গ্রহীতারা কোথায় অপেক্ষা করে?

$\begin{array}{lll}\text { অপেক্ষা করার স্থান: } & \text { श্যাঁ } & \text { ना } \\ \text { নির্ধারিত কক্ষে } & \text { ১ } & 2 \\ \text { বারান্দায় } & \text { ১ } & 2 \\ \text { খোলা জায়গায় } & \text { ১ } & 2 \\ \text { সেবাদাতার কক্ষে } & \text { ১ } & 2\end{array}$

8 । সেবাকেন্দ্রে পানির উৎস কি?

ট্যাপর পানি
টিউবওয়েল
তোলা পানি
কোন পানির ব্যবস্থা নেই

$\begin{array}{ll}\text { शाँ } & \text { ना } \\ 3 & 2 \\ 3 & 2 \\ 3 & 2 \\ 2 & 2\end{array}$


Q। কেন্দ্রে সেবাদাতার হাতটোয়ার কি ব্যবস্থা আছে?

शों, ট্যাপের পানির সাহায্যে
হাं, টিউবওয়েল পানির সাহায্যে
তোলা পানির সাহায্যে
কোন ব্যবস্থা নেই

$\begin{array}{ll}\text { इाँ } & \text { ना } \\ 3 & 2 \\ 3 & 2 \\ 3 & 2 \\ 2 & 2\end{array}$

৬। শৌচাগারের অবস্থা কি?

পরিচ্ছন্ন
মোটামুটি পরিচ্ছন্ন
নোংড়া
থুবই নোংড়া
কোন শৌচাগার নেই

$\begin{array}{ll}\text { शाँ } & \text { ना } \\ 2 & 2 \\ 2 & 2 \\ 2 & 2 \\ 3 & 2 \\ 3 & 2\end{array}$

৭। এই ক্রিনিকে কি পি, ভি (PV Examination) পরীক্ষা করা হয়?

$\begin{array}{ll}\text { शाँ } & \text { 2 } \\ \text { ना } & \text { 2 }\end{array}$

৮। পি, ভি পরিক্ষার (PV Examination) জন্য কি কোন পৃথক কক্ষ আছ?

$\begin{array}{ll}\text { इ্যাঁ পৃথক কক্ষ } & \text { ग } \\ \text { इ্যা একই কক্ষ আলाদা জয়গা } & 2 \\ \text { नाই } & 0\end{array}$

৯। পি, ভি পরীক্ষা কোথায় করা হয়?

রোগী পরীক্ষার টেবিলে

ও, টি, টেবিলে ।

১০। পি, ভি পরীক্ষার জন্য আলোর উৎস কি ?

$\begin{array}{ll}\text { স্পট লাইট } & \text { ১ } \\ \text { টর্চলাইট } & 2 \\ \text { স্বাভাবিক আলো } & \checkmark\end{array}$

১১। পি, ভি পরীক্ষার জন্য নিম্নলিখিত যন্ত্রপাতিগুনো কি আছে এবং কতটা আছে ?

Vaginal Cuscos
Sim's Vaginal Speculum
Sponge holding forceps
Instrument tray
Lifter
Spot light / Torch light
Other

$\begin{array}{llr}\text { श्यों } & \text { ना } & \text { मशच्या } \\ 2 & 2 & - \\ 2 & 2 & - \\ 2 & 2 & - \\ 2 & 2 & - \\ 2 & 2 & - \\ 3 & 2 & - \\ 3 & 2 & -\end{array}$

১২। জীবানু মুক্ত করনের জন্য সেবা কেন্দ্রে কোন কোন ধরনের ব্যবহার উপয়াগী যন্ত্রপাতি রয়েছে?

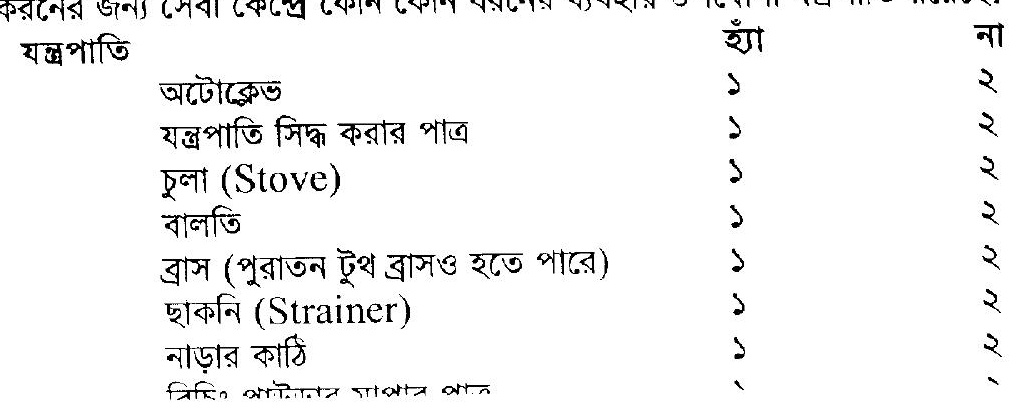


১৩। নীচের কোন কোন সামগ্রী সংক্রমণ (Infection) প্রতিরোধের জন্য সেবা কেন্দ্রে সরবরাহ পাওয়া যায়? সামগ্রী

ব্রিচিং পাউডার
গুড়ো সাবান
বার সাবান
গ্লাভস
তুলা
জ্বানানি (কেরোসিন)
ডেটল/স্যাভলন
আয়োডিন
স্পিরিট
ক্রোরোহেব্সিডিন/হিবিসল

$\begin{array}{ll}\text { शाँ } & \text { ना } \\ 2 & 2 \\ 3 & 2 \\ 3 & 2 \\ 3 & 2 \\ 2 & 2 \\ 3 & 2 \\ 2 & 2 \\ 2 & 2 \\ 3 & 2 \\ 2 & 2\end{array}$

১8। পরীক্ষার সময় কি পর্দা/গোপনীয়তা (Visual Privacy) বজায় থাকে? ( কোথায় PV করা হয় দেখুন, বুঝুন তারপর লিখুন)

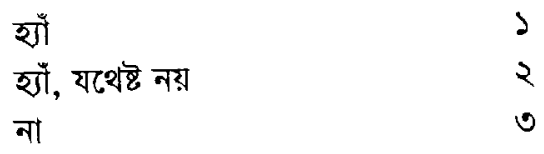

১৫। রোগের ইতিহাস নেয়ার সময় কথা বার্তা বলার গোপনীয়তা (Auditory Privacy) কি বজায় থাকে?

राँ

হॉॉ কিছুটা থাকে
$>$

2

১৬। চিকিৎসা বিষয়ক বর্জ (Waste) কি ভাবে পরিত্যাক্ত হয়?

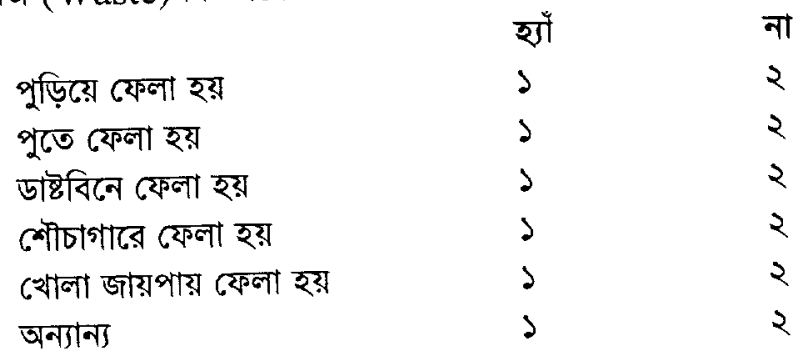




\section{Appendix 5}

\section{STRENGTHENING RTI/STD SERVICES IN H\&FWCs \\ Population Council, I haka}

আপনি বা আপনার স্বামী/স্ত্রী বা আপনার যুবতী মেয়ে কি নীচের শারীরিক সমস্যাগুলোতে ভুগছেন?

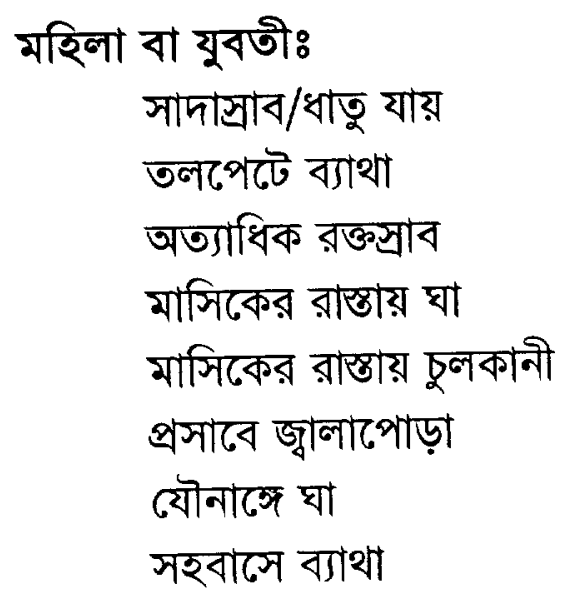

$$
\begin{aligned}
& \text { এলেঙ্গাঁ - বৃহুপ্তিবার } \\
& \text { নারানদিয়া - রবিবার } \\
& \text { পাইকরা - মঙ্গলবার }
\end{aligned}
$$

ইউনিয়ন পরিবার কল্যান কেন্দ্র 


\section{Appendix 6}

\section{STRENGTHENING RTI/STD SERVICES IN H\&FWCS}

Population Council. Dhaka

\section{রেফারেল ফরম}

1. রেজি: নং

2. সেবা প্রহীতার নামঃ

3. পরিবার কল্যান কেন্দ্রের নামঃ এলেঙা -1 , নারানদিয়া -2 , সহদেবপুর -3 , পাইকরা -4

4. রোগের বিবরণঃ

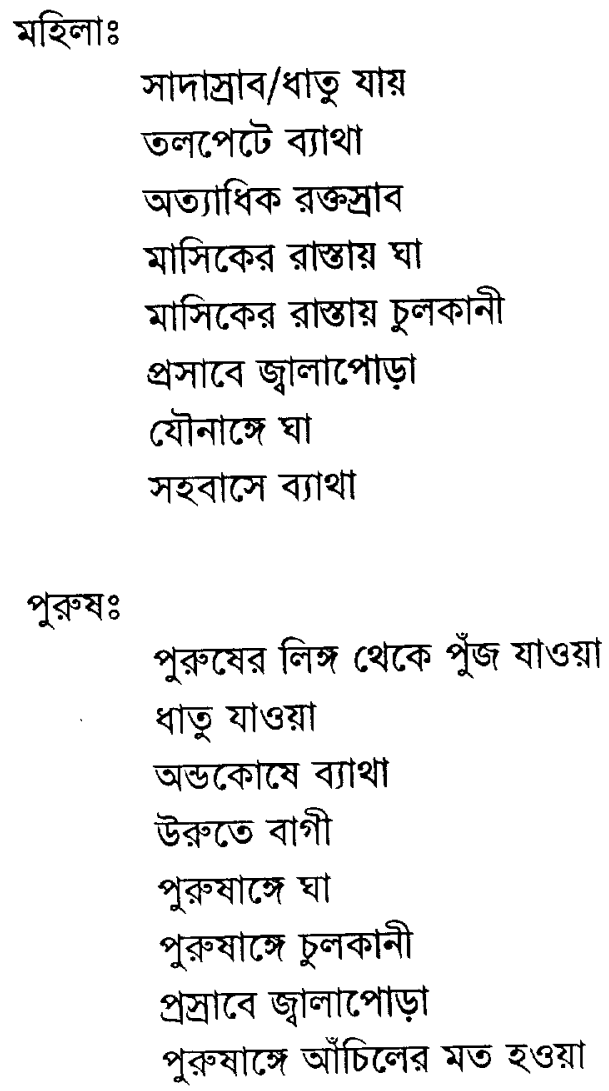

5. রেফার করা হলোঃ

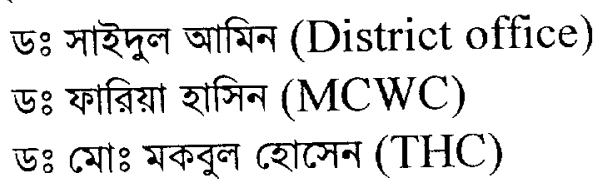




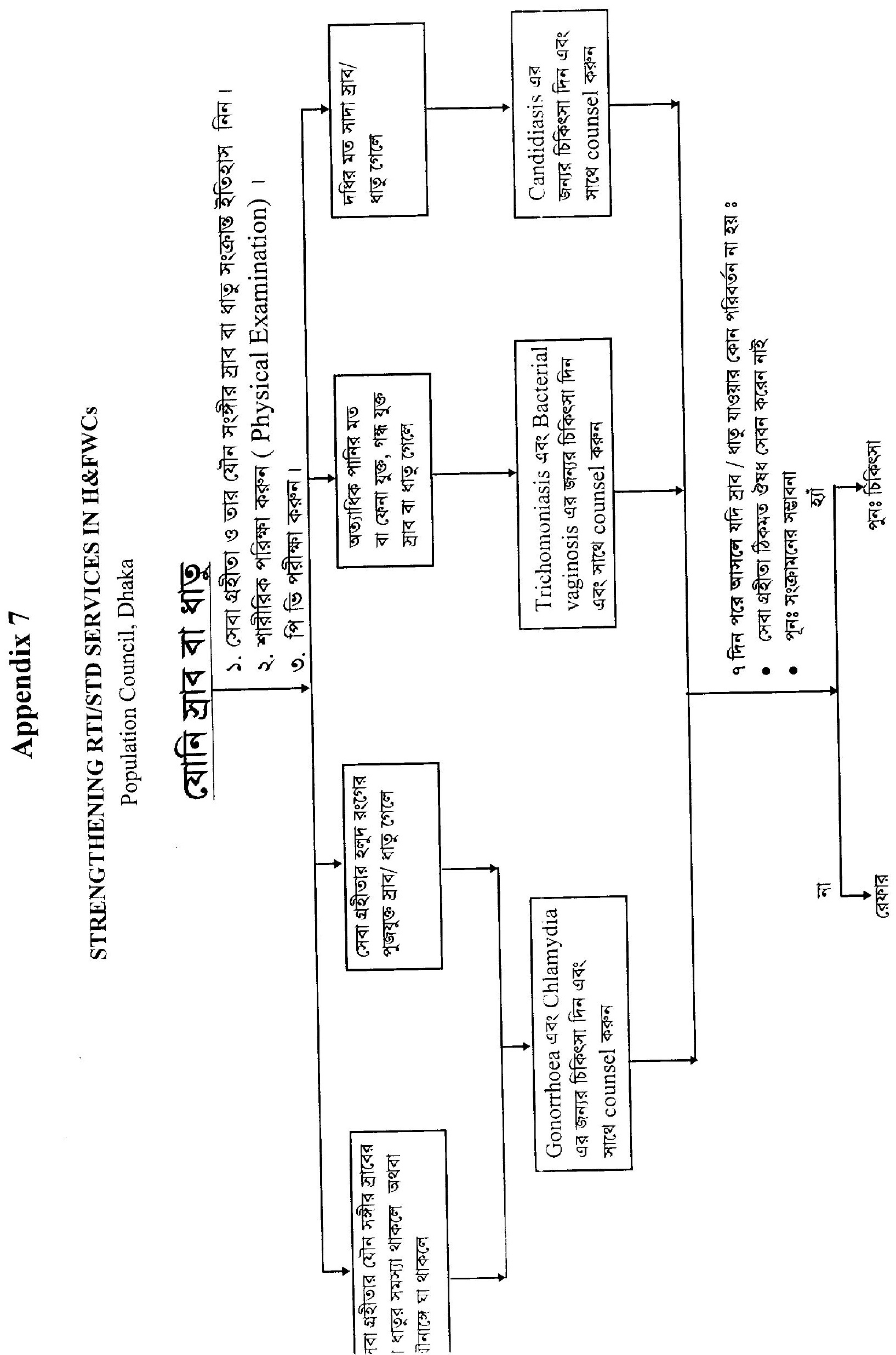




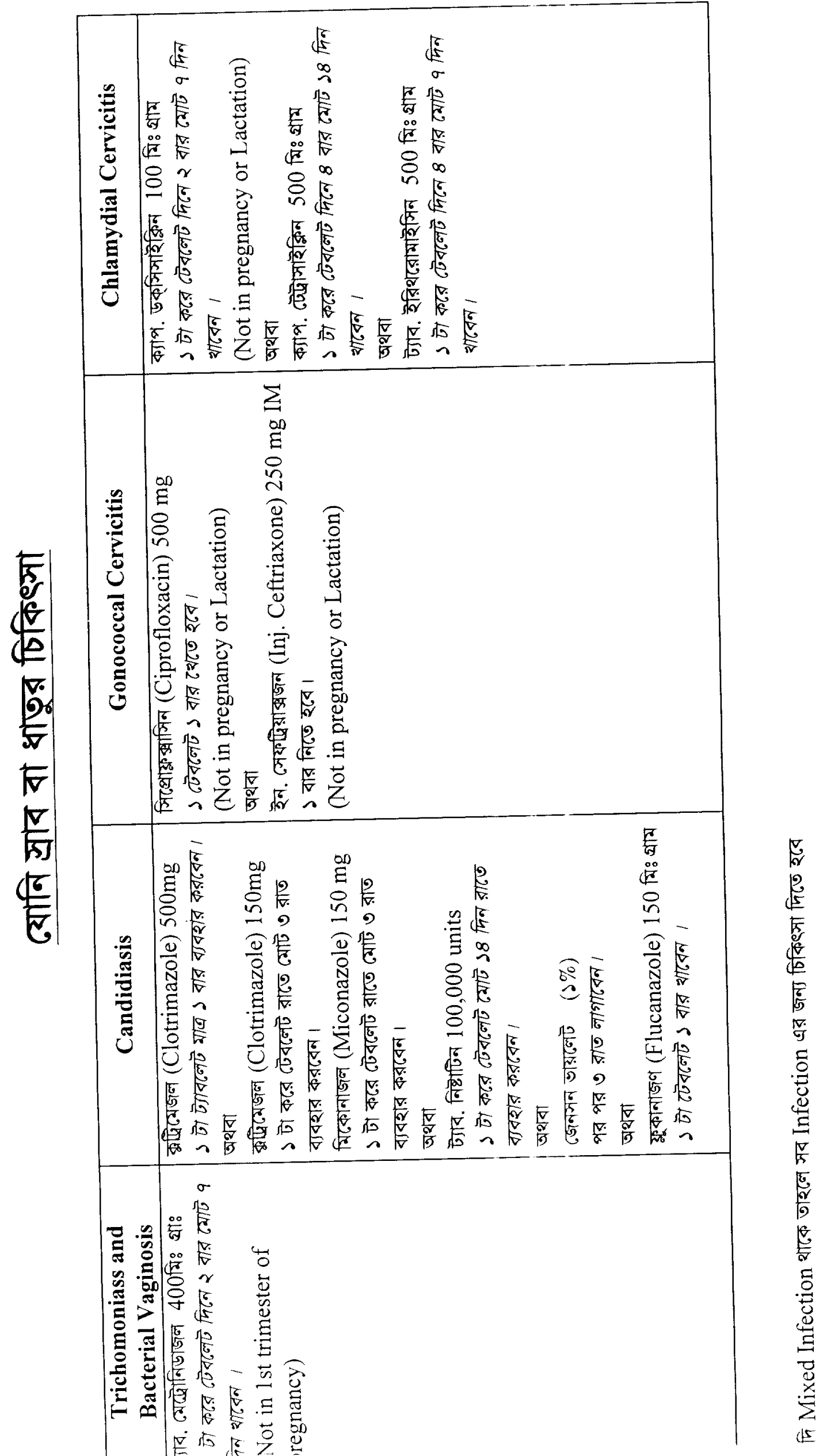




\section{Appendix 8}

\section{STRENGTHENING RTI/STD SERVICES IN H\&FWCs \\ Population Council, Dhaka}

\section{(Client Profile)}

SL. No.

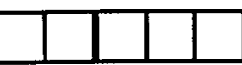

1. রেজি: নং (GH-general client, FP-Family Planning client, RT-RTI client, AN-ante natal, PN- post natal, MR-MR client)

2. তারিখঃ

3. পরিবার কল্যান কেন্দ্রের নামঃ এলেঙ্গ / নারানদিয়া / পাইকরা / সহদেবপুর

4. সেবাদান কারীর নামঃ এফ, ডব্লিউ, ভি (FWV) / সাকন্মে (SACMO) /

এম, ও (এম, সি, এইচ - এফপি) / এম ও (র্লিনিক)

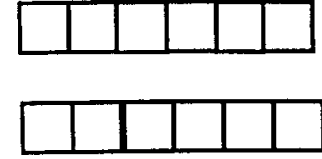

অन्্ान्य:

5. সেবা গ্রহীতার নামঃ
a. বয়সঃ
বৎসর
b. স্বামী / পিতার নামঃ
c. ইউनिয়নঃ
d. গ্যাম:
e. বাড়ীঃ

6. আপনি কি বিবাহিতা?

হাঁ / না / প্রযোজ্য নয়
a. স্বামীর সাথে থাকেন (বিবাহিত হলে )?
হॉা / ना / প্রযোজা নয়

7. আপনি কোন ক্ষাস পর্যন্ত লেখাপড়া করেছেন?

ক্লাস

8. আপনার স্বামী কত ক্লাস পর্যন্ত পড়াঙ্ৰনা করেছেন?

ক্লাস

9. আপনি কি ধরনের কাজ করেন?

10. আপনার স্বামী কি ধরনের কাজ করেন?
চাকুরী / দিন মজুরী / ছাত্রী / গৃহিণী / কিছু করি না / অনান্য

গ্রানে চাকুরী / শহরে চাকুরী / বিদেশে চাকুরি / গ্রামে ব্যবসা / শহরে ব্যবসা / কৃষি/ দিনমজুর / ট্রাক চালক / কার চালক / রিক্সা চালক বা ভ্যান চালক / কিছু করি না , প্রত্যাজ্য নয় / অন্যান্যঃ

11. আপনার শারীরিক কি কি অসুবিধার জন্য আজ আপনি এখানে এসেছেন?
a. সাদাস্রাব/ধাতু যায়
b. তলপেটে ব্যাথা
c. অত্যাধিক রক্তস্রাব
d. মাসিকের রাস্তায় ঘা
e. মাসিকের রাস্তায় চুলকানী
f. প্রসাবে জ্বালাপোড়া
g. बयोनाऊ घा
h. সহবাসে ব্যাথা
i. অन्यान्यः (এখানে যারা অন্য जেবা গ্রহন করতে এসে RTI ধরা পড়ড়ছে তাদেরকে লিপিবদ্ধ করুন) 
12. সাসিকের ইতিহাসः
a. মাসিকের ধরনঃ
निয়মিত / अनिয়মিত
b. কত বৎসর বয়সে আপনার মাসিক হরু হয়েছিল ?
c. কত দিন আগে আপনার শেষ মাসিক হয়েছিল? দিन
d. আপনার মাসিক কতদিন পরপর হয়?
e. মাসিক হলে তা কতদিন থাকে? দিन
f. মাসিকের সময় দিনে কতবার মাসিকের কাপড় বা টিপা বদলাতে হয়?
g. মাসিকের সময় কি আপনার তলপেটে ব্যথা হয় ? বার
h. আপনার শেয মাসিকের সময় মাসিকের রক্ত কি গন্ধযুক্ত ছিল? হ্যা / না

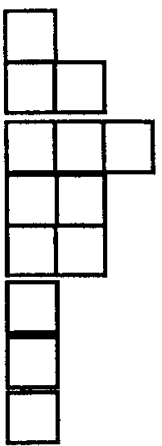

\section{3. গর্ভের ইতিহাসঃ (অবিবাহিতের বেলায় প্রজোয্য নয়)}

a. কত বৎসর বয়সে আপনার বিবাহ হয়েছিল ? (সম্পূর্ন বৎসরের সংখ্যা লিখুন) বঙসর

b. কত বৎসর বয়সে আপনার প্রথম সন্তানের জন্ম হয়েছিল ? (সম্পূর্ন বৎসরের সংখ্যা লিখুন ) ব९সর

c. এখন আপনার নিজের কতজন জীবিত সন্তান আছে? सन

d. আপনার সর্বশেষ প্রসব কি রকম ছিল?

স্বাভাবিক প্রসব / অস্ত্রপ্রচারের মাধ্যনে প্রসব / যন্ত্রপাতির সাহায্যে / কাঁচা যাওয়া

(Spontaneous Abortion)/ গর্ভপাত (Induced Abortion)

e. আপনার সর্বশেষ প্রসব কোথায় হয়েছিল?

নিজ বাসায়/সেবাদানকারীর বাসায় / প্রাইভেট ক্লিনিকে / এফ, ডাব্লিউ, সি তে / হাসপাতাল

f. আপনার সর্বশেষ প্রসবের সময় কে সেবাদান করেছিল ?

ডাক্তার / এফ, ডাব্লিউ, ভি / নার্স / টি, বি, এ / অয়ীয় / গ্রামের দাই / পল্লি চিকিৎসক

\section{4. শারীরিক তথ্যঃ}
a. সাধারণ স্বাস্থ্যঃ
ভাল / মাটামুটি / খারাপ
b. তাপমাত্রা ফাঃ / সেঃ
c. রক্তশূন্যতাঃ
আছে / নাই
d. জিহ্বা বা গালে घा
আছে / নাই

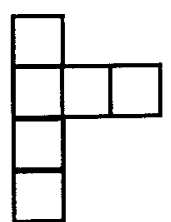

15. আপনার কি অস্বাভাবিক ধাতু বা সাদস্রাব যায়? হযাঁ / না
a. কত দিন ধরে ধাতু বা সাদাস্রাব যাচ্ছে? দিন/মাস/বছর
b. ধাতু বা স্রাব যাওয়ার ধরন কি? মাঝে মাঝে / সবসময়

c. ধাতু কখন বেশী যায় ? মাসিকের পর পর / মাসিকের মাঝামাঝি সময় / মাসিক ফরু হওয়াার আগে

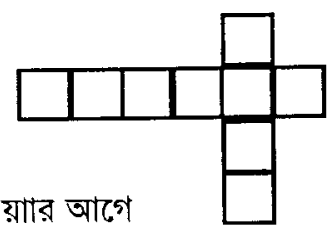

d. ধাতু বা স্রাব যাওয়ার পরিমান কি রকম? অল্প / মাঝামাঝি (কোন কাপড় বা টিপা ব্যবহার করতে হয় না)/ খুব বেশী পরিমান (কাপড় বা টিপা ব্যবহার করতে হয়) / প্রযোজ্য নয়

e. ধাতুর বা স্রাবের রং কি রকম? পানির মত / দই এর মত সাদা / হনুদ রঙের / ফেনাযুক্ত / লালছে / প্রযোজ্য নয়

f. ধাতু বা স্রাব কি গঙ্ধ যুক্ত ? গक্ধ আছছ / গঞ্ধ নাই / জানিনা / শ্যোজ্য নয় 
17. আপনার যৌনাঞ্সে কি ঘা আছে?

আছে / নাই
a. यদি থাকে, তবে আপনার এই ঘা থেকে কোন রক্ত বা পুঁজ যায় কি?
হ্যা / না / প্রযোজ্য নয়

18. আপনার কি সহবাসের সময় ব্যাথা হয়?

হ্যাঁ, যৌনাঙ্গের মধ্যে ব্যথা হয় বা জ্বালাপোড়া হয় / হাঁ, তলপেটে ব্যাথা

হয় / হাঁ, শারীরিক অসুবিধা নাগে/ কোন ব্যাথা হয় না / প্রযোজ্য নয়

19. আপনার কি তলপেটে ব্যাথা আছে?

আছে / নাই

a. যদি থাকে, তবে ব্যাথাটির ধরন কি রকম?

খুব বেশী ব্যথা (সাধারন কাজ কর্ম করতে পারিনি) / ব্যাথা আছে, তরে সাধারন কাজ কর্ম করতে বেশ অসুবিধা হয় / অল্প অল্প ব্যথা, তবে সাধারন কাজ কর্ম করতে পারি / প্রযোজ্য নয়

20. আপনার কি প্রস্রাবের সময় জ্বালাপোড়া হয়?

श्याँ / ना

20a. আপनाর কি घन ঘন প্রস্রাব হয়?

श्याँ / ना

21. আপনি কি আমাশয় ভুগছেন?

छौौ / ना

22. আপনার সমস্যাগুলোর জন্য গত ছয় মাসের মধ্যে কি কখনো চিকিৎসা করিয়েছেন? করে থাকলে কতদিন আগে এবং কার কাছ থেকে করিয়েছেন?

\begin{tabular}{|c|c|c|c|c|c|c|}
\hline $\begin{array}{l}\text { গত ছয় মালের মধ্যে চিকিৎসা করে } \\
\text { থাকলে নিচের কোন্ সমস্যাণ্োর } \\
\text { জন্য কর্রিয়েছেন, টিক চিহ্ন দিন । }\end{array}$ & 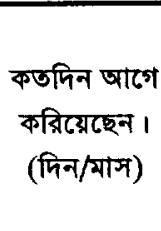 & 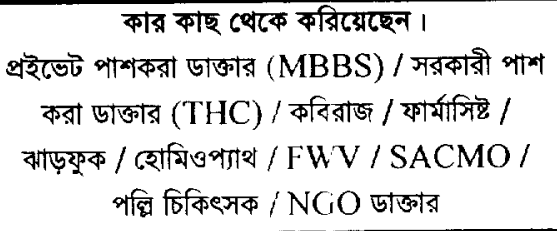 & & & & \\
\hline a. সাদাস্রাব/ধাতু যায় & & & & & & \\
\hline b. অলপেটে ব্যাথা & & & & & & \\
\hline c. অত্যাধিক রক্তস্রাব & & & & & & \\
\hline d. মাসিকের রাস্তায় ঘা & & & & & & \\
\hline e. মাসিকের রাস্তায় চৃলকানী & & & & & & \\
\hline f. প্রসাবে জ্বালাপোড়া & & & & & & \\
\hline g. যৌনাঞ্গে ঘা & & & & & & \\
\hline h. সহবাসে ব্যাথা & & & & & & \\
\hline i. অন্যান্য: & & & & & & \\
\hline
\end{tabular}

23. নীচের সমস্যা গুলোর মধ্যে কোন কোন টি আপনার স্বামীর যৌনাগে গত ছয় মাসের মধ্যে হয়েছে? সমস্যা যদি হয়ে থাকে চিকিৎসা করিয়েছেন কি এবং চিকিৎসা করিয়ে থাকলে, কার কাছ থেকে এবং কতদিন আগে করিয়েছেন?

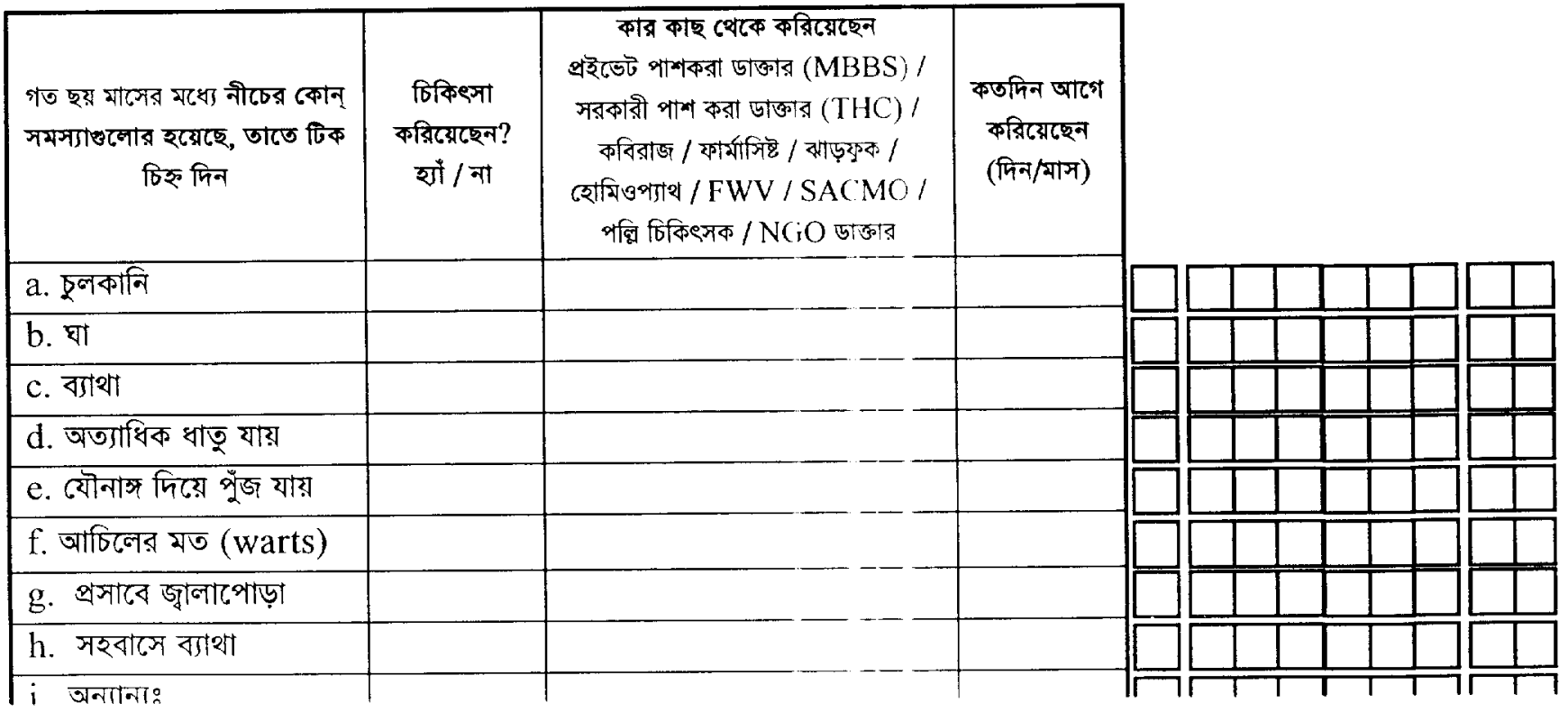


24. আপনি কি বর্তমানে কোন পরিবার পরিকল্পনা পদ্ধতি ব্যবহার করেন?

হ্যা / না / প্রযোজ্য নয়

a. হ্যা হলে, নীচের কোন পদ্ধতি ব্যবহার করেন? বড়ি / কনডম / ইনজেক্শন / আই, ইউ, ডি / নরপ্নান্ট / লাইগেশন / ভেসেক্টমি / সনাতন পদ্ধতি (নিদিষ্ট করুন) / প্রযোজ্য নয়

b. কত দিন যাবৎ ব্যবহার করেন দিन/ মাস / বছর

25. তলপেট পরিক্ষাঃ
a. স্পর্শ কাতরতা (Tenderness)
b. পেটের চাকা (Abdominal Mass)
কম / মাবামাঝি / খুব বেশী / নাই
ছোট / মাঝারি / বড় / নাই

পি, ভি পরীক্ষা :

26. Genital region (খালি চোখে পরীক্ষা)ঃ
a. রং পরিবর্তন (discolouration)
আছে / নাই
b. প্রদাহ (Inflammation)
আছে / নাই
c. ফুলা (Swelling)
d. গোটা (Nodule)
আছে / নাই
e. घा (Ulcer)
f. স্রাব
ব্যাথা যুক্ত / ব্যাথা বিহীন / নাই
आছে / নাই
আছে / নাই

27. Speculum দিয়ে পরীক্ষা : (গর্ভ বতীর বেলায় প্রযোজ্য নয়)
a. সারভিক্র :
স্বাভাবিক ( Healthy) / রক্তাভ (Reddish) / নীলাভ (Bluish) / প্রদাহ
(Inflammation) / ফ़লा (Swelling) / क्षয় (old tear) / घा (Ulcer)
। প্রযোজ্য নয়
a1. যোনী পথ (Vaginal wall)
প্রদাহ (Inflammation) , ফুলা (Swelling) / ঘा (Ulcer) / ফুস্কা
(Papilla) / প্রয়াজ্য নয়
b. স্রাবঃ
আছে / নাই /প্রযোজ্য নয়
b1. স্রাবের উৎস :
b2. স্রাবের রং:
b3. স্রাবের পরিমানঃ
b4. স্রাবের গহ্ধঃ
ভেজাইনাল / সারভাইক্যাল (Cervical) / ইউরেথ্রাল / প্রযোজ্য নয়
পানির মত / দইই এর মত সাদা / হলদে / সবুজাভ / রক্তয়ক্ত / প্রযোজ্য নয়
সামান্য / মোটামুটি / খুববেশী / প্রযোজ্য নয়
b5. স্রাবের ঘনত্ঃঃ
স্বাভাবিক / দুর্গদ্ধযুক্ত / প্রয়াজ্য নয়
পাতলা / ঘন / প্রযোজ্য নয়

28. বাই-ম্যানুয়েল ইক্সামিনেশন (Bi-manual Examination)
a. সারভিক্সের সচলতা (Cervical Mobility)
b. সারভিক্সের স্পর্শকাতরতা(Cervical Tenderness)
c. ফরনিক্সের স্পর্শকাতরতা (Fornix Tenderness)
d. ফরনিক্সের Adhesion
e. বারথোলিন গ্রান্ডের (Bartholin Gland)
স্পর্শ কাতরতা (Tenderness)
সচল আছে / সচল নাই / প্রযোজ্য নয় আছে / নাই / প্রযোজ্য নয় আছে / নাই / প্রযোজ্য নয় ফ্রি (Free) / দৃঢ़ হওয়া (Fixed) / প্রযোজ্য নয় আছে / নাই / প্রযোজ্য নয়

29. তাৎক্ষুনিক রোগ নির্ণয় (Provisional Diagnosis)
a. ক্যান্ডিডিয়াসিস (Candidiasis)
b. ট্রাইকোরোনিয়াসিস (Trichomoniasis)
c. ব্যাক্টিরিয়াল ভেজোনাসিস (Vacterial Vaginosis)
d. গনোরিয়া (Gonorrhoea)
e. ক্লামাইডিয়া (Chlamydia)
f. পি, আই, ডি (P. I. D)
g. সারভিসাইটিস (Cervicitis)
h. গর্ভবতী (Pregnant)
i. जनान्य: 
30. তাৎক্ষনিক ব্যবস্থা(Management)
a. চিকিৎসা দেওয়া হলো (হ্যা হনে, a1 নং প্রশ্ন প্রযোজ্য)
b. উপদেশ দেওয়া হলো (खौঁ হলে, b1 নং প্রশ্ন প্রযোজ্য)
c. রেফার করা হনো (হ্যাঁ হলে, c1 নং প্রশ্ন প্রযোজ্য)

a1. কি কি চিকিৎসা দেয়া হলো?

a1 1. ট্যাব. মেট্রোনিডাজল (Tab. Metronidazole) 400মিঃ গ্রাঃ

১ টা করে টেবলেট দিনে ২ বার হোট ৭/১৪ দিন খাবেন ।

a1_2. জেনসन ভায়লেট(Gentian Violet) (১\%)

ক্ষত জায়গায় পর পর ৩ রাত লাগাবেন / ক্ষত জায়গায় দিনে ২ বার মোট ৩ সপ্তাহ লাগাবেন।

a1 3. ক্যাপ. ডক্সিসাইক্লিন (Cap. Doxycycline) 100 মিঃ গ্রাম

১ টা করে ক্যাপসুল দিনে ২ বার নোট ৭/১৪ দিন খাবেন ।

a1_4. ক্যাপ. টেট্রাসাইক্ষিন (Cap. Tetracycline) 500 মিঃ ज্রাম

১ টা করে ক্যাপসুল দিনে 8 বার মোট ৭/১৪ দিন খাবেন।

al_5. ট্যাব. ইরিথরোমাইসিন (Tab. Erythromycine) 500 মিঃ গ্রাম

১ টা করে টেবলেট দিনে 8 বার মোট ৭/১৪ দিন খাবেন।

al 6. ট্যাব. নিষ্টাটিন (Tab. Nystatin)

১টা করে টেবনেট মোট ১৪ দিন রাতে ব্যবহার করবেন।

a1_7. ক্লোট্রিমাজল (Clotrimazole) 150 মিঃ গ্রাম

১ টা করে রাতে মোট ৩ রাত ব্যবহার করবেন ।

a1 8. মিকোনাজল (Miconazole) $150 \mathrm{mg}$

১ টা করে টেবলেট রাতে মোট ৩ রাত ব্যবহার করবেন ।

a1 9. সিপ্রোফ্লক্সাসিন (Ciprofloxacin) 500 মিঃ গ্রাম

১ টা টেবলেট ১ বার খেতে হবে।

a1_10. ইন. সেফটিয়াক্সজন (Inj. Ceftriaxone) $250 \mathrm{mg} \mathrm{IM}$

১ বার নিতে হরে।

a1 11. কেট্রেইমক্সাজল (Cotrimoxazole)

২ টা করে টেবলেট দিনে ২ বার বমাট ৭ দিন খাবেন ।

al 12. ফ্রকনাজল (Flucanazole) 150 মিঃ গ্রাম

১ টা টেবলেট ১ বার খাবেন ।

a1_13. কেনামাইসিन (Kanamycin) 25 मিঃ গ্রাম/কেজি IM

১ বার নিতে হবে।

a1_14. সিরাপ ইরিথ্রোমাইসিন (Erythromycin Syrup) 50 মিঃ গ্রাম/কেজি

দিনে 8 বার মোট ১৪ দিন খেতে হরে।

b1. কি কি উপদেশ দেওয়া হলো?

b1 1. সামান্য লবন গরম পানি দিয়ে যৌনাঙ ধুয়ে ফেলুন।

b1 2. মাসিকের কাপড় পরিস্কার করে ষুয়ে রোদে শুকিয়ে ব্যবহার করবেন

b1_3. মল ত্যাগের পর মলদ্বার পরিস্কার করার সময় কখনো নীচের দিক থেকে উপরের দিকে পরিস্কার করবেন না (মহিলাদের বেলায়)।

bl 4. ভিনেগারের ডুশ নিবেন (Pregnant মহিলাদের বেলায়)।

b1 5. সুস্থ না হওয়া পর্যত্ত থ্যৌন সঙ্ থেকে বিরত থাকবেন অথবা যৌন সঙরের সময় Condom ব্যবহার করবেন।

b1_6. আপনার স্বামী / যৌন সঙ্গীকে ক্রিনিকে নিয়ে আসবেন।

b1_7. আপনার স্বামী / যৌন সঙ্গীর কোন উপসর্গ না থাকনেও র্চিকৎসা করাতে হবে।

b1 8. আপনি এবং আপনার স্বামী / যৌন সঙী দু‘জনে একনাথে ঔষধ খেতে হবে।

bl_9. উপসর্গ চলে গেলেও, ঔষধের পুরো মেয়াদ পূর্ন করটেন

b1_10. যৌনাজ্যে ব্যবহারের জন্য দেওয়া ঔষধ সঠিক নিয়ের ব্যবহার করবেন।

bl_11. ৩ দিন/৭ দিন পরে Follow up এ আসতে হৃব 
c1. নং প্রশ্ন হ্যা হলন, কোথায় রেফার করা হলো? এম, সি, ডাব্রিউ, সি (MCWC) / থানা স্বাস্থ্য কমপ্নেক্স (THC) / অ্রযোজ্য নয়

\section{1. ফनো আপ ভিজিট (Follow up visit)}

a. প্রথম ভিজিটঃ

b. তারিখঃ

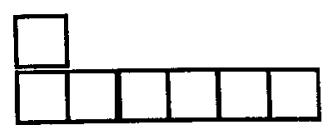

c. সম্পর্ন চিকিৎসা নেওয়া (Treatment Compliance)

সম্পূর্ন চিকিৎসা নিয়েছে (Full Course) / আংশিক চিকিৎসা নিয়েছে (Partial Course) /

কোন চিকিৎসা নেয়নি (No Treatment)

d. বর্তমান শারীরিক অবস্থা (Prognosis Status)

সম্পূর্ন আরোগ্য লাভ করেছে / আংশিক আরোগ্য লাভ করেরে / আরোগ্য লাভ করেনি / অবস্থার অবনতি হুয়েছে

e. ব্যবস্থা/ চিকিৎসা (Management/Treatment)

e1. চিকিৎসা চালিয়ে যাওয়া

e2. ঔষধ পরিবর্তন করে আবার চিকিৎসা দেওয়া

e3. রেফার করা

e4. উপদেশ দেওয়া

a1. দ্বিতীয় ভিজিটঃ

b1. তারিখঃ

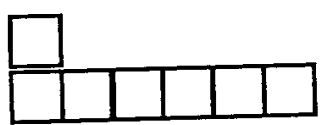

c1. সম্পুর্ন চিকিৎসা নেওয়া (Treatment Compliance)

সম্পূর্ন চিকিৎসা নিয়েছে (Full Course) / আংশিক চিকিৎসা নিয়েছে (Partial Course) /

রোন চিকিৎসা নেয়নি (No Treatment)

d1. বর্ত্ा শারীরিক অবস্থা (Prognosis Status)

সম্পূর্ন আরোগ্য লাভ করেছে / আংশিক আরোগ্য লাভ করেছে / আরোগ্য লাভ করেনি / অবস্থার অবনতি হয়েছে

el. ব্যবস্থা / চিকিৎসা (Management/Treatment)

el_1. চিকিৎসা চালিয়ে যাওয়া

el_2. ঔষধ পরিবর্তন করে আবার চিকিৎসা দেওয়া

el_3. রেফার করা

e1_4. উপদেশ দেওয়া 


\section{Appendix 9}

\section{CONTENTS OF DDS AND SATELLITE KIT}

\begin{tabular}{|c|c|c|c|}
\hline DDS kit & Quantity & Satellite kit & Quantity \\
\hline $\begin{array}{l}\text { 1. Ampicillin caps } 250 \mathrm{mg} \text { strips } \\
\text { pack/1000 }\end{array}$ & 2 & & \\
\hline $\begin{array}{l}\text { 2. Ampicillin oral susp. } \\
125 \mathrm{mg} / 5 \mathrm{ml} \text {, box } / 25 \mathrm{X} 100 \mathrm{ml}\end{array}$ & 4 & $\begin{array}{l}\text { 1. Ampicillin pwdw/oral susp. } \\
125 \mathrm{mg} / 5 \mathrm{ml}, 60 \mathrm{ml}\end{array}$ & 50 \\
\hline $\begin{array}{l}\text { 3. Tetracycline HCL OPH OINT. } \\
1 \% \text { box/50X5g tubes }\end{array}$ & 1 & & \\
\hline $\begin{array}{l}\text { 4. Chlorhexidine Conc. Soln. } 20 \% \\
\text { box } / 5 \mathrm{X} 100 \mathrm{ml}\end{array}$ & 1 & & \\
\hline 5. $\quad$ Salbutamol Tab 4mg 100 & 2 & & \\
\hline $\begin{array}{l}\text { 6. Methylergometrine Tab } 0.125 \mathrm{mg} \\
\text { blst/strp pack/100 }\end{array}$ & 1 & $\begin{array}{l}\text { 2. Ferrous salt }+ \text { folic acid Tab. } \\
60+0.25 \mathrm{mg} 1000\end{array}$ & 20 \\
\hline $\begin{array}{ll}\text { 7. Ferrous salt }+ \text { folic acid Tab. } \\
60+0.25 \mathrm{mg} 1000\end{array}$ & 5 & & \\
\hline $\begin{array}{l}\text { 8. Metronidazole tab 400mg } \\
\text { box/4X1000 }\end{array}$ & 1 & $\begin{array}{l}\text { 3. Metronidazole tab } 250 \mathrm{mg} 1000 \\
\text { 4. Metronidazole oral susp } \\
200 \mathrm{mg} / 5 \mathrm{ml}, 100 \mathrm{ml}\end{array}$ & $\begin{array}{c}4 \\
50\end{array}$ \\
\hline 9. $\quad$ Paracetamol Tab 500mg 1000 & 2 & 5. Paracetamol Tab 500mg 1000 & 3 \\
\hline $\begin{array}{l}\text { 10. Paracetamol elixir } 125 \mathrm{mg} / 5 \mathrm{ml} \text {, } \\
\text { box } / 25 \times 60 \mathrm{ml}\end{array}$ & 2 & $\begin{array}{l}\text { 6. Paracetamol elixir } 125 \mathrm{mg} / 5 \mathrm{ml} \text {, } \\
\text { box } / 25 \mathrm{X} 60 \mathrm{ml}\end{array}$ & 100 \\
\hline $\begin{array}{l}\text { 11. Sulfamethoxazole }+ \\
\text { trimethoprim tab } 400+80 \mathrm{mg} 500\end{array}$ & 1 & $\begin{array}{l}\text { 7. Sulfamethoxazole }+ \\
\text { trimethoprim tab } 400+80 \mathrm{mg} 500 \\
\text { 8. Sulfamethoxazole }+ \\
\text { trimethoprim tab } 100+20 \mathrm{mg} 100\end{array}$ & $\begin{array}{l}5 \\
5\end{array}$ \\
\hline $\begin{array}{l}\text { 12. Retinol caps high potency } \\
200,000 \mathrm{IU} \text { pack/100 }\end{array}$ & 3 & & \\
\hline $\begin{array}{l}\text { 13. Benzyl benzoate saponated conc. } \\
\text { Soln. } 90 \% 1 \mathrm{~L}\end{array}$ & 1 & $\begin{array}{l}\text { 9. Benzyl benzoate lotion 25\% 1L } \\
\text { btl }\end{array}$ & 6 \\
\hline $\begin{array}{l}\text { 14. Chloramphenicol eye/ear drop } \\
0.5 \% \text { box } / 25 \mathrm{X} 10 \mathrm{ml}\end{array}$ & 2 & & \\
\hline $\begin{array}{l}\text { 15. Hyoscine butyl bromide tab } \\
10 \mathrm{mg} \mathrm{pack} / 100\end{array}$ & 2 & & \\
\hline $\begin{array}{l}\text { 16. Benzoic + salicylic acid } \\
\text { oint } / \text { cream } 6 \%+3 \% 1 / 2 \mathrm{~kg}\end{array}$ & 2 & $\begin{array}{l}\text { 10. Benzoic + salicylic acid } \\
\text { oint/cream } 6 \%+3 \% 1 / 2 \mathrm{~kg}\end{array}$ & 1 \\
\hline 17. Gentian violet pwdr $25 \mathrm{~g}$ & 1 & 11. Gentian violet pwdr $25 \mathrm{~g}$ & 1 \\
\hline $\begin{array}{l}\text { 18. Plaster adhesive zinc oxide } \\
75 \mathrm{~mm} \text { X 5m roll }\end{array}$ & 3 & & \\
\hline $\begin{array}{l}\text { 19. Envelopes for tablets } \\
\text { polyethylene pkt of } 100\end{array}$ & 10 & $\begin{array}{l}\text { 12. Envelopes for tablets } \\
\text { polyethylene pkt / } 10\end{array}$ & 150 \\
\hline $\begin{array}{l}\text { 20. Albendazole tab } 200 \mathrm{mg} \\
\text { pack/100 }\end{array}$ & 3 & & \\
\hline $\begin{array}{l}\text { 21. Penicillin V tab } 250 \mathrm{mg} \text { (strips) } \\
\text { pack/1000 }\end{array}$ & 2 & & \\
\hline \multirow[t]{4}{*}{ 22. Vitamin B complex tab pack/100 } & 2 & & \\
\hline & & 13. Mebendazole tab $100 \mathrm{mg} 100$ & 15 \\
\hline & & $\begin{array}{l}\text { 14. Nystatin pessaries } 100000 \mathrm{IU} \\
\text { pack of } 15\end{array}$ & 30 \\
\hline & & $\begin{array}{l}\text { 15. Alum. Hydrox } 405 \mathrm{mg}+\text { magn. } \\
\text { Hydrox. } 100 \mathrm{mg} \text { pack } / 1000\end{array}$ & 1 \\
\hline
\end{tabular}




\section{Appendix 10}

\section{CONTENTS OF NEW DDS KIT}

\begin{tabular}{|c|c|}
\hline Name of Medicine & Quantity \\
\hline 1. Cap. Amoxycillin. $250 \mathrm{mg}$., blister/strip pack & 1000 cap \\
\hline 2. Susp. Amoxycillin (Dry syrup) $125 \mathrm{mg} / 5 \mathrm{ml}, 100 \mathrm{ml}$ botl & 50 botl \\
\hline 3. Tab. Salbutamol, $4 \mathrm{mg} / \mathrm{Tab}$, strip/blister of 10 tabs. & 50 tab. \\
\hline $\begin{array}{l}\text { 4. Tab. Hyoscine Butyl Bromide, } 10 \mathrm{mg} \text { strip/blister of } 10 \\
\text { tabs. }\end{array}$ & 10 strip/blister. (100 tabs) \\
\hline 5. Tab. Methyl Ergometrine, $0.125 \mathrm{mg} /$ botl. of 30 tabs. & 1 botl. $(30 \mathrm{tab})$ \\
\hline $\begin{array}{l}\text { 6. Ferrous salt } 60 \mathrm{mg}+\text { Folic Acid } 0.25 \mathrm{mg} \text { tab., } 1000 \text { tab. in } \\
\text { one container. }\end{array}$ & 8 container $(8000 \mathrm{tab})$ \\
\hline $\begin{array}{l}\text { 7. Tab. Metronidazole, } 400 \mathrm{mg} / \mathrm{tab} .1000 \mathrm{tab} \text {. in one } \\
\text { container }\end{array}$ & 1 container (1000 tab) \\
\hline 8. Tab. Paracetamol, $500 \mathrm{mg} / \mathrm{tab} .1000$ tab in one container & 1 container $(1000$ tab) \\
\hline 9. Syp. Paracetamol, $125 \mathrm{mg} / 5 \mathrm{ml}$, botl of $60 \mathrm{ml}$ & 25 botl \\
\hline $\begin{array}{l}\text { 10. Tab. Sulphamethoxazole+Trimethoprim, } 400 \mathrm{mg}+80 \\
\text { mg, Pack of } 100 \text { tabs. }\end{array}$ & 8 pack (800 tab.) \\
\hline $\begin{array}{l}\text { 11. Tab. Sulphamethoxazole+Trimethoprim, } 100 \mathrm{mg}+20 \mathrm{mg} \text {, } \\
\text { Pack of } 100 \mathrm{tab} \text {. }\end{array}$ & 10 pack (1000 tab.) \\
\hline $\begin{array}{l}\text { 12. Benzyl Benzoate. Saponated Conc. soln, } 90 \%, 1000 \mathrm{ml} \\
\text { in one container }\end{array}$ & 2 container \\
\hline 13. Gentian Violet Powder, 25 G/bottle & 1 bottle \\
\hline 14. Tab. Chlorpheniramine Maleate, $4 \mathrm{mg} / \mathrm{tab}, 100 \mathrm{tab} / \mathrm{pack}$ & 3 pack $(300 \mathrm{tab})$ \\
\hline $\begin{array}{l}\text { 15. Benzoic Acid+Salicylic Acid Oint. } 6 \%+3 \% \text { Pks of } 500 \\
\text { G in one container. } 1 \text { container }\end{array}$ & 1 container \\
\hline 16. Tab. Albendazole, $400 \mathrm{mg} / \mathrm{tab}, 100$ tab./botl & 2 botl. (200 tab.) \\
\hline $\begin{array}{l}\text { 17. Tab./Cap. Vitamin B complex (Thiamine } \mathrm{Hcl}=5 \mathrm{mg} \text {, } \\
\text { Pyridoxine } \mathrm{Hcl}=2 \mathrm{mg} \text {, Riboflavin=2mg and } \\
\text { Nicotinamide }=20 \mathrm{mg} \text { ) } 1000 \text { in one container }\end{array}$ & 1 (one) 1000 tabs. \\
\hline $\begin{array}{l}\text { 18. Tab. Antacid, Al. Hydroxide } 250 \mathrm{mg}+\text { Mag. Hydroxide } \\
400 \mathrm{mg} / \mathrm{tab} . \text {, blister/strip of } 10 \text { tabs. }\end{array}$ & 100 strip / blister (1000 tab.) \\
\hline 19. Ointment-Neomycin/Bacitracin Eye/Ear & 25 tubes \\
\hline $\begin{array}{l}\text { 20. Envelope-Dispensing } 10 \mathrm{~cm} / 7 \mathrm{~cm} \text { with flap made of } 36 \\
1 \mathrm{bs} \mathrm{KPM}\end{array}$ & $800 \mathrm{pcs}$ \\
\hline
\end{tabular}

